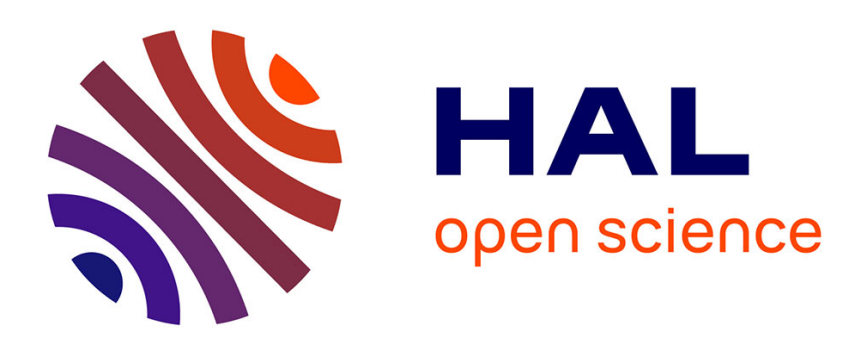

\title{
Instability of dielectrics and conductors in electrostatic fields
}

Grégoire Allaire, Jeffrey Rauch

\section{To cite this version:}

Grégoire Allaire, Jeffrey Rauch. Instability of dielectrics and conductors in electrostatic fields. Archive for Rational Mechanics and Analysis, 2017, 124, pp.233-268. hal-01321566

\section{HAL Id: hal-01321566 https://hal.science/hal-01321566}

Submitted on 26 May 2016

HAL is a multi-disciplinary open access archive for the deposit and dissemination of scientific research documents, whether they are published or not. The documents may come from teaching and research institutions in France or abroad, or from public or private research centers.
L'archive ouverte pluridisciplinaire HAL, est destinée au dépôt et à la diffusion de documents scientifiques de niveau recherche, publiés ou non, émanant des établissements d'enseignement et de recherche français ou étrangers, des laboratoires publics ou privés. 


\title{
INSTABILITY OF DIELECTRICS AND CONDUCTORS IN ELECTROSTATIC FIELDS
}

\author{
GRÉGOIRE ALLAIRE ${ }^{1}$ AND JEFFREY RAUCH ${ }^{2}$
}

\begin{abstract}
This article proves most of the assertion in $\S 116$ of Maxwell's treatise on electromagnetism. The results go under the name Earnshaw's Theorem and assert the absence of stable equilibrium configurations of conductors and dielectrics in an external electrostatic field.
\end{abstract}

Key words: Instability, electromagnetism, dielectric, perfect conductor, Dirichlet's Theorem, Earnshaw's Theorem.

2010 Mathematics Subject Classification: 70H14, 35B35, 35Q60, 35Q61, 35Q70, 70E91.

${ }^{1}$ Centre de Mathématiques Appliquées, UMR CNRS 7641, École Polytechnique, Université Paris-Saclay, 91128 Palaiseau, France.

Email: gregoire.allaire@polytechnique.fr

2 Department of Mathematics, University of Michigan, Ann Arbor 48109 MI, USA.

Email: rauch@umich.edu

\section{INTRODUCTION}

This paper presents rigorous proofs of theorems that go under the name of Earnshaw's Theorem in Electrostatics. The original assertion dates to 1842. The generalisations that we discuss are introduced in the remarkable $\S 116$ of Maxwell's Treatise on Electromagnetism [11]. The simplest version is that a point charge in the electrostatic field of other fixed charges that are at finite distance from the point charge cannot be in a position of stable equilibrium. We begin with an overview of the correct assertions of earlier authors, contrasting them with dynamical instability.

1.1. What did Earnshaw prove? Earnshaw's paper [6] discusses possible structures of the aether. He proposes that particles of the aether when subjected to a small displacement should oscillate, with oscillation independent of the direction of the displacement. The analysis is at the level of what is today called the theory of small oscillations. For an equilibrium $\underline{x}$ of the dynamical equations

$$
x^{\prime \prime}=-\nabla_{x} V(x), \quad \nabla_{x} V(\underline{x})=0 .
$$


expand the potential energy

$$
V(x)=V(\underline{x})+\frac{1}{2} \sum_{i, j} \frac{\partial^{2} V(\underline{x})}{\partial x_{i} \partial x_{j}}(x-\underline{x})_{i}(x-\underline{x})_{j}+\text { h.o.t } .
$$

The theory of small oscillations omits the higher order terms thereby linearizing equation (1.1). Solutions of the linearized equation are all sinusoidal oscillations if and only if the hessian of $V$ at $\underline{x}$ is strictly positive definite. This in turn implies that $V$ has a strict local minimum.

If all interparticle forces are inverse square law forces as is the case in electrostatics, then the potential is a harmonic function. Earnshaw proves the following.

Proposition 1.1. For a harmonic potential, it is impossible that the solutions of the linearized equation at an equilibrium are all oscillatory.

Proof. If the solutions are all oscillatory then the hessian is strictly positive. Then

$$
\Delta V(\underline{x})=\operatorname{Tr}[\operatorname{Hess} V(\underline{x})]>0 .
$$

In particular, $V$ is not harmonic.

For Earnshaw this implied that looking for force laws governing the aether he needed to look beyond inverse square law forces. From the perspective of Maxwell's $\S 116$, when $V$ is harmonic then except degenerate cases, the linearized equation will have exponentially growing modes indicating instability.

1.2. What did Maxwell prove? The fascinating $\S 116$ of Maxwell's treatise has three different arguments related to Earnshaw's Theorem. The first two prove assertions with the flavor of instability, and the third gives a number of far reaching generalisations. The proof of most of them is the subject of this paper.

1.2.1. Repulsive restoring force. Maxwell considers a test particle of charge 1 near an equilibrium $\underline{x}$ of the potential $V(x)$ created by other charges that do not move. The restoring force is equal to the electric field $E(x)=-\nabla V(x)$. Since there are no charges near $\underline{x}, V$ is harmonic on a neighborhood of $\underline{x}$ and Gauss' Theorem asserts that the flux of the electric field through small spheres centered at $\underline{x}$ is equal to zero.

Proposition 1.2. Suppose that $\underline{x}$ is an equilibrium for (1.1) and the potential energy $V$ is a non constant harmonic function on a neighborhood of $\underline{x}$. Then there are arbitrarily small displacements $y$ so that the force $E$ exerted at $\underline{x}+y$ has projection on $\mathbb{R} y$ pointing away from $\underline{x}$, that is $y \cdot E(\underline{x}+y)>0$. The force pushes away from $\underline{x}$.

Proof. Maxwell only proves the weaker assertion $y \cdot E(\underline{x}+y) \geq 0$ as follows. Changing coordinates we may suppose that the equilibrium $\underline{x}$ is equal to 0 . If the weaker assertion 
were false, one could choose a small $r>0$ so that for all $\mathbf{e}$ with $\|\mathbf{e}\|=1, \mathbf{e} \cdot E(r \mathbf{e})<0$ and so that $V$ is harmonic on the ball of radius $r$ centered at $\underline{x}$. Integrating over the sphere of radius $r$ yields a strictly negative flux of $E$ through the sphere of radius $r$. By Gauss' theorem that flux vanishes. The contracdition completes Maxwell's proof.

To prove the stronger assertion it suffices to show that it is impossible that $y \cdot E(y)$ vanishes identically on a neighborhood of the origin. The function $y \cdot E(y)$ is real analytic on the largest charge free ball $B$ centered at the origin. If it vanishes on a neighborhood of 0 , then it vanishes identically on $B$. Expand in a Taylor series of terms $E_{j}$ homogeneous of degree $j$,

$$
E=E_{1}+E_{2}+\cdots+E_{j}+\cdots, \quad E \cdot y=E_{1} \cdot y+E_{2} \cdot y+\cdots+E_{j} \cdot y+\cdots .
$$

The right hand equation is the Taylor expansion of $E(y) \cdot y$. Since $E(y) \cdot y=0$ it follows that for all $j, E_{j}(y) \cdot y=0$. From the Taylor expansion

$$
V=V_{0}+V_{1}+V_{2}+\cdots+V_{j}+\cdots
$$

one finds that $E_{j}=-\nabla V_{j+1}$. Therefore

$$
\nabla V_{j}(y) \cdot y=-E_{j-1}(y) \cdot y=0 .
$$

The Euler homogeneity relation implies that $\nabla V_{j}(y) \cdot y=j V_{j}$. Therefore $V_{j}=0$ for $j \geq 1$ proving that $V$ is constant on $B$.

1.2.2. No minima of potential energy. Another argument in Maxwell and other classics depends on the maximum principal asserting that nonconstant harmonic functions cannot have local extrema. Since the electrostatic potential energy $V(x)$ is harmonic it cannot have a local minimum. It is very common to confuse the notions of stability of an equilibrium and minimum of potential energy. This is based on an appealing but incorrect dynamical intuition. If there are nearby points $x_{n}$ with $V\left(x_{n}\right)<V(\underline{x})$ then a particle starting near $\underline{x}$ will seek to decrease its potential energy by moving toward these points.

The counterexample of Wintner [19] based on the classical construction of Painlevé [13] and recalled in our earlier article [16], gives a smooth potential $V$ and an equilibrium $\underline{x}$ that is not a local minimum and nevertheless is stable. The states $x_{n}$ are inaccessible from states near $\underline{x}$. They are blocked by maxima of potential energy between $x_{n}$ and $\underline{x}$ serve as barriers.

1.2.3. Maxwell's more general assertions. Maxwell's $\S 116$ proves a variety of more general statements about minima of potential energies. He first considers a solid body $K$ with attached charges and placed in the field of fixed external charges at finite distance from the body. The restoring force argument works without modification when applied to the center of mass of the body. There are small translations $y$ of the body for which the force has projection on $y$ pointing away from $\underline{x}$. 
Proposition 1.3. Suppose that there is an equilibrium with the center of mass at $\underline{x}$. Denote by $V(x)$ the potential energy of $K$ translated so that the center of mass is moved to $x \approx \underline{x}$. Then if $V(x)$ is not constant there are arbitrarily close translates with strictly lower potential energy.

Proof. The potential energy $V(x)$ is a harmonic function. If it is not equal to a constant it cannot have a local minimum.

Example 1.4. A classical result of Newton [12] in 1687 asserts that the gravitational field of a uniformly mass distribution on the surface of a sphere vanishes in the interior. Exactly the same argument applies to the electric field of a uniform spherical charge distribution. Therefore a charged body $K$ inside a uniformly charged sphere is acted on by no forces. This is a nontrivial example where the potential energy $V$ is locally constant.

Maxwell then considers the more complicated case that the rigid body is a perfect conductor or a dielectric.

Corollary 1.5. If the solid body $K$ is a charged or neutral perfect conductor or a dielectric and there is an equilibrium with center of mass at $\underline{x}$. Denote by $V_{1}(x)$ the potential energy of the body with its charges frozen in their equilibrium configuration on $K$ and then translated with center of mass at $x$. Denote by $V(x) \leq V_{1}(x)$ the potential energy of the translated body with center of mass at $x$ that minimizes energy with charges free to move. If $V_{1}(x)$ is not constant, then there are arbitrarily small translations that lower the potential energy.

Proof. If the harmonic function $V_{1}$ is not constant then there are arbitrarily close points $x$ with $V_{1}(x)<V_{1}(\underline{x})=V(\underline{x})$. The potential energy $V(x)$ is obtained as a minimum over charge distributions on $K$ in the conductor case and over polarizations in the case of a dielectric. The frozen configuration is one of the admissible states, so $V(x) \leq V_{1}(x)<$ $V(\underline{x})$.

Remark 1.6. Maxwell goes on to prove similar assertions when some of the external charges are fixed and others lie on perfect conductors or dielectrics. The argument is the same. First the external charges are frozen in their equilibrium condition, then they are left to seek their configuration minimizing, hence lowering, the potential energy.

1.3. Duffin's Theorem. Duffin [4] proved the following important result concerning the potential energy of conductors and dielectric. It allows one to give a strengthening of Corollary 1.5. His proof is for external charge distributions with finite self energy. The method of renormalized energy in $\S 10$ allows one to remove this hypothesis.

Theorem 1.7. $V(x)$ from Corollary 1.5 satisfies $\Delta V(x) \leq 0$. In particular if $V(x)$ is non constant, then it cannot have a local minimum. 
1.4. Dynamics, Dirichlet, and Lyapunov. The striking thing about all of these interesting results is that not a single one addresses the question of dynamic stability, that is the stability of an equilibrium as understood by Lyapunov and Poincaré and recalled in the next definition. For more details on stability, instability and rigid body dynamics see for example [2].

Definition 1.8. i. A point $\bar{x}$ is an equilibrium of (1.1) if and only if $\nabla_{x} V(\bar{x})=0$, equivalently $\bar{x}$ is a stationary point of $V$.

ii. Such an equilibrium $\underline{x}$ is stable when for any $\epsilon>0$ there is $a \delta>0$ so that the solution of (1.1) with initial condition satisfying $\|x(0)-\bar{x}(0)\|^{2}+\left\|x^{\prime}(0)\right\|^{2}<\delta^{2}$ exists for all positive time and satisfies for all $t>0,\|x(t)-\bar{x}(0)\|^{2}+\left\|x^{\prime}(t)\right\|^{2}<\epsilon^{2}$.

Example 1.9. If the potential energy $V$ is constant on a neighborhood of $\underline{x}$, then there are no forces and the equilibrium is unstable since a particle starting at the equilibrium with a very small velocity moves steadily away.

A result due to Dirichlet after earlier work by Lagrange is the following.

Theorem 1.10. If the equilibrium $\bar{x}$ is a strict local minimum of $V$ then it is stable.

The classic instability criterion is due to Lyapunov.

Theorem 1.11. If at an equilibrium the hessian of $V$ has a strictly negative eigenvalue, then the equilibrium is unstable.

1.5. Kozlov's Theorem. Kozlov [9] in 1987 proved the following result that implies that a point charge in an electrostatic field cannot have a stable equilibrium (see [18] for our favorite proof).

Theorem 1.12. Suppose that $\underline{x}=0$ is an equilibrium for (1.1) and that the Taylor expansion of $V$ at the origin is

$$
V=V_{m}(x)+\text { higher order terms }
$$

with $V_{m}$ a not identically zero homogeneous polynomial of degree $m \geq 2$ and that $V_{m}$ does not have a minimum at the origin. Then the equilibrium is unstable.

Example 1.13. Kozlov's Theorem implies instability of a point particle at equilibrium in an electrostatic field. Indeed, If $V$ is an electrostatic potential then it is harmonic. If it is constant Example 1.9 proves instability. If it is not constant then it has an expansion as in Theorem 1.12 with $V_{m}$ a non zero harmonic polynomial of degree $m$. Thus $V_{m}$ does not have a local minimum at the origin by the maximum principal for harmonic functions. Theorem 1.12 implies instability. 
1.6. Instability for a charged rigid body remains open. The argument of Kozlov does not imply the instability of the motion of a charged rigid body with fixed charges. An example of such a body is the small equilateral triangle in the figure centered at the origin and with equal negative charges at the vertices. It is in the field of three equal

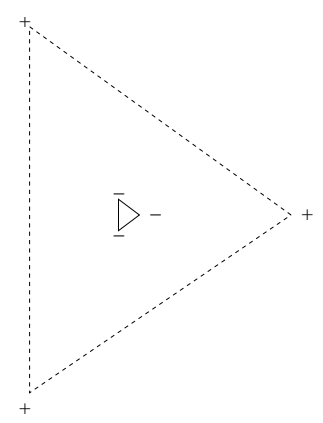

positive charges at the vertices of a concentric equilateral triangle. Consider only motions of the triangle in its own plane. The position of the triangle is defined by the position $x \in \mathbb{R}^{2}$ of the center and the angle $\theta \in S^{1}$ of rotation about the center. The triangle at the origin and with $\theta=0$ is in equilibrium. A straightforward but not short computation shows that the potential energy $V(x, \theta)$ has Taylor expansion at the origin

$$
V(x, \theta)=V(0,0)+\frac{V_{\theta, \theta}(0)}{2} \theta^{2}+O\left(|x, \theta|^{3}\right), \quad V_{\theta, \theta}(0)>0 .
$$

The leading term has a minimum at $\theta=0$ so Kozlov's Theorem does not apply. The expansion also implies that $V$ is not the solution of second order linear homogeneous elliptic partial differential equation (see [16]).

1.7. Palamodov's outline of proof. Lyapunov conjectured that if $V$ is real analytic and $\underline{x}$ is an equilibrium that is not a local minimum of $V$ then the equilibrium is unstable. This was also on Arnold's 1976 list of problems [1]. See [10], [14], [17] for the case $d \leq 2$. In 1995 Palamodov [15] outlined a very interesting proof. A detailed proof has never been published. If this conjecture were proved it would imply our instability theorems and also the instability of charged rigid bodies that we do not prove. Our proof for dielectrics and perfect conductors yields a physical description of the instability mechanisms. It has much to offer even if Lyapunov's general conjecture gets a complete proof.

1.8. Our instability dichotomy. Our main new results are Theorems 7.1 and 11.5 that prove the instability of rigid bodies that are either dielectrics or conductors. The same argument works as soon as the body has an open subset that has strictly positive polarizability or is perfectly conducting. Of the general assertions of Maxwell the only one that escapes our analysis is the case of a rigid body with fixed charges. 
Suppose that $V(x)$ is the potential energy of the body translated by $x$ as in Corollary 1.5. Duffin's theorem asserts that $\Delta V \leq 0$. Assume now that $\underline{x}$ is an equilibrium position of the body. We prove II of the following remarkable dichotomy.

I. If $\Delta V(\underline{x})<0$ then the smallest eigenvalue $\lambda$ of the hessian with respect to $x$ at $\underline{x}$ is strictly negative. The smallest eigenvalue of the hessian with respect to all the variables is $\leq \lambda<0$. Theorem 1.11 implies instability.

II. If $\Delta V(\underline{x})=0$, then, amazingly, the field of the external charges is constant on a neighborhood of $K$. In this case all small translates of $K$ are also equilibria. The motion of $K$ with initial position equal to the equilibrium and translating with constant small speed are solutions that escape from the equilibrium. Those solutions prove instability.

Remark 1.14. i. In $\S 10, \S 11$, we extend Duffin's results to external charge distributions with infinite self energy. In particular the classic example of external point charges.

ii. The conductor case is a limiting case of the dielectric case but instability is not necessarily inherited by limits. For example, the equilibrium $\underline{x}=0$ with

$$
V^{\epsilon}(x)=\epsilon x^{2}+x^{3}, \quad \text { resp. } \quad V^{\epsilon}(x)=\epsilon x^{3}+x^{4}
$$

is stable (resp. unstable) for $\epsilon>0$ and unstable (resp. stable) when $\epsilon=0$.

The dielectric case is presented first. The case of conductors begins in $\S 8$.

\section{RIGID BODY DYNAMICS AND THE EUCLIDEAN GROUP}

This section recalls some of the elements of Euler's brilliant geometrization of rigid body dynamics. The compact set $K \subset \mathbb{R}^{3}$ is called the body. It is endowed with a finite positive Borel measure $\mu$ with $\mu(A)=\int_{A} d \mu$ giving the mass in $A$. The total mass if equal to $\mu\left(\mathbb{R}^{3}\right)$.

2.1. Motions and the euclidean group. A motion is a differentiable curve $[a, b] \ni$ $t \mapsto \gamma(t) \in \operatorname{Euc}(3)$, where $\operatorname{Euc}(3)$ denotes the Euclidean group of orientation preserving isometric affine transformations. The position of $K$ at time $t$ for this motion is $\gamma(t) K$. The point $k \in K$ moves on the path $\gamma(t) k$. The velocity at time $t$ of the point $\gamma(t) k$ is $d(\gamma(t) k) / d t$.

2.2. Tangent space $T_{I}(\operatorname{Euc}(3))$. For $v \in \mathbb{R}^{3}$ denote by $M(v) \in \operatorname{Euc}(3)$ the translation operator $M(v) x:=x+v$. The map $v \mapsto M(v)$ is a group isomorphism of $\mathbb{R}^{3}$ onto the three dimensional translation subgroup of $\operatorname{Euc}(3)$. The one parameter subgroup $t \mapsto M(t v)$ has tangent vector at $t=0$ that we identify with $v$. In this way the set of such $v \in \mathbb{R}^{3}$ is identified with a three dimensional subspace of the tangent space $T_{I}(\operatorname{Euc}(3))$. Similarly, for $\omega \in \mathbb{R}^{3} \backslash 0$ define an antisymmetric real linear transformation $A_{\omega} \in \operatorname{Hom}\left(\mathbb{R}^{3}\right)$ by $A_{\omega} x:=\omega \wedge x$. Then $t \mapsto e^{t A_{\omega}}$ is a one parameter subgroup of Euc(3). Its infinitesimal 
generator is $A_{\omega}$ that we associate to $\omega$. The map $\omega \mapsto A_{\omega}$ yields a second three dimensional subspace of $T_{I}(\operatorname{Euc}(3))$. These two subspaces generate the tangent space that we therefore identify with the set of pairs $(v, \omega)$.

2.3. A mass density on $K$ defines a Riemannian metric on $\operatorname{Euc}(3)$. The total kinetic energy at time $t$

$$
\begin{aligned}
\text { Kinetic Energy } & =\frac{1}{2} \int_{K}\left\|\frac{d \gamma(t) k}{d t}\right\|^{2} d \mu(k) \\
& =\frac{1}{2} \int_{K}(\gamma(t) k)^{\prime} \cdot(\gamma(t) k)^{\prime} d \mu(k):=\frac{1}{2} g_{\gamma(t)}\left(\gamma^{\prime}(t), \gamma^{\prime}(t)\right) .
\end{aligned}
$$

This defines a candidate scalar product $g_{\gamma(t)}$ on the tangent space $T_{\gamma(t)}(\operatorname{Euc}(3))$.

Replacing $\gamma(t) k$ by $E \gamma(t) k$, for any $E \in \operatorname{Euc}(3)$, does not change the value of $g$ in the above formula. The candidate metric is left invariant.

There are degenerate cases where this does not define a Riemannian metric. If the support of $\mu$ is contained in a line, then rotations about the line have zero kinetic energy so the candidate metric is not positive definite. The next result shows that this is the only exception.

Proposition 2.1. If the support of $\mu$ is not contained in a straight line then for all $\beta \in \operatorname{Euc}(3), g_{\beta}$ is strictly positive definite on the tangent space $T_{\beta}(\operatorname{Euc}(3))$.

Proof. By left invariance, $g_{\beta}$ is strictly positive for all $\beta$ if and only if $g_{I}$ is strictly positive on $T_{I}(\operatorname{Euc}(3))$. Therefore it suffices to prove that if $g_{I}$ is not strictly positive, then the support of $\mu$ is contained in a line.

Suppose that $\Gamma \in T_{I} \backslash 0$ and $g_{I}(\Gamma, \Gamma)=0$. If $\Gamma$ is associated to $(v, \omega)$, then $\gamma(t)=$ $M(t) e^{A_{\omega} t}$ is a curve with $\gamma(0)=I, \gamma^{\prime}(0)=\Gamma$, and $\gamma^{\prime}(0) k=v+\omega \wedge k$. Therefore $g_{I}(\Gamma, \Gamma)=0$ holds if and only if

$$
\int(v+\omega \wedge k) \cdot(v+\omega \wedge k) d \mu(k)=0 .
$$

If $\omega=0$ then $\Gamma \neq 0 \Rightarrow v \neq 0$ so the integrand is strictly positive. Therefore (2.1) cannot hold since $0 \leq \mu$ is not identically equal to zero.

For $\omega \neq 0$, decompose $v=v_{\|}+v_{\perp}, v_{\|}=\|\omega\|^{-2}(v, \omega) \omega$. The Pythagorean Theorem yields $\|v+\omega \wedge k\|^{2}=\left\|v_{\|}\right\|^{2}+\left\|v_{\perp}+\omega \wedge k\right\|^{2} \geq\left\|v_{\|}\right\|^{2}$. Thus (2.1) can only hold when $v_{\|}=0$. In that case, write $v_{\perp}=-\omega \wedge \zeta$ for a unique $\zeta \perp \omega$. Then (2.1) reads

$$
\int\|\omega \wedge(k-\zeta)\|^{2} d \mu(k)=0 \text {. }
$$

Therefore $(k-\zeta) \| \omega$ for all $k \in \operatorname{supp} \mu$. Equivalently, for all $k \in \operatorname{supp} \mu, k \in\{\zeta+\mathbb{R} \omega\}$. This proves that $\operatorname{supp} \mu$ is a subset of the line $\zeta+\mathbb{R} \omega$. 
Remark 2.2. i. In the degenerate case, the natural configuration space is the quotient of Euc(3) by the subgroup that leaves the points of $\operatorname{supp} \mu$ fixed. We leave to the reader the straight forward modifications needed to treat that degenerate case.

ii. The principle of stationary action implies that the motion of the rigid body $K$ in the absence of external forces follows geodesics of the Riemannian manifold $\{\operatorname{Euc}(3), g\}$.

Definition 2.3. A $C^{1}$ curve $\zeta(t)$ in a Riemannian manifold $(\mathcal{R}, g)$ is parametrized proportional to arclength when $g_{\zeta(t)}\left(\zeta^{\prime}(t), \zeta^{\prime}(t)\right)=$ constant $>0$.

Proposition 2.4. i. For any body $K$ and mass measure $\mu$ satisfying the hypothesis of Proposition 2.1, the translation subgroup of Euc(3) is isometric to $\mathbb{R}^{3}$ with the scalar product equal to $\mu\left(\mathbb{R}^{3}\right) / 2$ times the standard Euclidean scalar product.

ii. The translation subgroup is totally geodesic. Moreover, for any $v \in \mathbb{R}^{3} \backslash 0$ the curve $t \mapsto M(t v)$ is a geodesic of Euc(3) parametrized proportional to arclength.

Proof. i. For $v \in \mathbb{R}^{3}$ denote by $M(v) \in \operatorname{Euc}(3)$ the operator translation by $v$. The map $v \mapsto M(v)$ is then a group isomorphism from $\mathbb{R}^{3}$ to the translation subgroup of $\operatorname{Euc}(3)$. It is an immediate consequence of the definition of the Riemannian metric on $\operatorname{Euc}(3)$ that this map preserves the Riemannian metrics so is an isometry.

ii. The curve $t \mapsto t v$ is a geodesic in $\mathbb{R}^{3}$ parametrized proportional to arclength with the metric from i. It image by the isometry $M$ is therefore a geodesic of $\operatorname{Euc}(3)$ parametrized proportional to arclength.

Example 2.5. Assertion ii is equivalent to the fact that the motion of the body $K$ given by $t \mapsto M(t v) K=K+t v$ is a solution of the equation of motion of rigid bodies in the absence of external forces. This is needed in the proof of instability in Case II of the dichotomy.

\section{Potential Energy of A Dielectric in AN EleCtrostatic Field}

Denote by $\dot{H}^{1}\left(\mathbb{R}^{3}\right)$ the homogeneous Sobolev space of distributions such that their first-order derivatives belong to $L^{2}\left(\mathbb{R}^{3}\right)$ and by $\dot{H}^{-1}\left(\mathbb{R}^{3}\right)$ its dual space.

Hypothesis 3.1. Suppose that $K$ is a compact connected lipschitzian body and that $\alpha \in$ $L^{\infty}(K)$ is a symmetric non negative matrix valued function on $K$. Suppose that $\alpha$ is strictly positive on a non empty open subset $K_{0} \subset K$. The function $\alpha$ is called the polarizability. Suppose that $\rho, q \in \dot{H}^{-1}\left(\mathbb{R}^{3}\right)$ with $\operatorname{supp} q \subset K$ and $K \cap \operatorname{supp} \rho=\varnothing$. The distribution $\rho$ describes the external charges while $q$ describes the charges fixed to $K$.

Notation. Denote by $n$ the unit outward normal to $K$ and by $\nu$ the unit outward normal to the complement $\Omega:=\mathbb{R}^{3} \backslash K$ as in the figure below. 


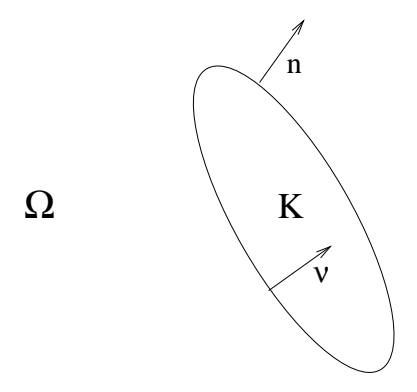

The electric field in the presence of the dielectric is the unique $E \in L^{2}\left(\mathbb{R}^{3}\right)$ such that

$$
\operatorname{curl} E=0, \quad-\operatorname{div} E=\rho+q+\operatorname{div} p, \quad p:=\alpha E .
$$

The vector valued function $p$ is called the polarization of the dielectric. The curl free condition is equivalent to $E=\nabla \psi$ for a unique $\psi \in \dot{H}^{1}\left(\mathbb{R}^{3}\right)$. That potential $\psi$ is the unique $\dot{H}^{1}\left(\mathbb{R}^{3}\right)$ solution of

$$
-\operatorname{div}(\epsilon \nabla \psi)=\rho+q, \quad \epsilon:=I+\alpha \geq I .
$$

The relation $E=\nabla \psi$ shows that $-\psi$ is the electrostatic potential energy. The quantity $\epsilon$ is called the dielectric permittivity. The solution $\psi$ is the unique minimizer in $\dot{H}^{1}\left(\mathbb{R}^{3}\right)$ of the functional

$$
\psi \quad \mapsto \quad \frac{1}{2} \int_{\mathbb{R}^{3}} \epsilon \nabla \psi \cdot \nabla \psi d y-\int_{\mathbb{R}^{3}} \psi(\rho+q) d y .
$$

Define the external field as the unique $E_{\text {ext }} \in L^{2}\left(\mathbb{R}^{3}\right)$ so that

$$
\operatorname{curl} E_{\text {ext }}=0, \quad \text { and, } \quad-\operatorname{div} E_{\text {ext }}=\rho .
$$

It is the field that would be present in the absence of $K$ with its charges $q$. Equivalently

$$
E_{\text {ext }}=\nabla \phi_{\text {ext }}, \quad \phi_{\text {ext }} \in \dot{H}^{1}\left(\mathbb{R}^{3}\right), \quad-\Delta \phi_{\text {ext }}=\rho .
$$

Proposition 3.2. The function $\psi$ is given by the formula

$$
\psi=\phi_{\text {ext }}-(\operatorname{div} \epsilon \nabla)^{-1}\left(q+\operatorname{div} \alpha E_{\text {ext }}\right) .
$$

Proof. Subtracting the equation for $E_{\text {ext }}$ from that determining $E$ yields

$$
\operatorname{curl}\left(E-E_{\text {ext }}\right)=0, \quad-\operatorname{div}\left(E-E_{\text {ext }}\right)=q+\operatorname{div} \alpha E .
$$

In the last, write $E=E-E_{\text {ext }}+E_{\text {ext }}$ to find

$$
\operatorname{curl}\left(E-E_{\text {ext }}\right)=0, \quad-\operatorname{div} \epsilon\left(E-E_{\text {ext }}\right)=q+\operatorname{div} \alpha E_{\text {ext }} .
$$

Writing $E-E_{\text {ext }}=\nabla \zeta$, yields

$$
-\operatorname{div} \epsilon \nabla \zeta=q+\operatorname{div} \alpha E_{\text {ext }}, \quad \zeta=(-\operatorname{div} \epsilon \nabla)^{-1}\left(q+\operatorname{div} \alpha E_{\text {ext }}\right),
$$

proving formula (3.3). 
Remark 3.3. i. The field $E-E_{\text {ext }}$ is uniquely determined by the values of $q$ and $\alpha E_{\text {ext }}$. ii. If two external charge distributions yield the same external field on $K$, then the fields $E-E_{\text {ext }}$ are identical. Therefore the fields on $K$ are identical. Therefore, the polarizations $\alpha E$ are identical.

Definition 3.4. The potential energy of the dielectric in equilibrium is defined as

$$
J:=-\frac{1}{2} \int_{\mathbb{R}^{3}}(\rho+q) \psi d y=\frac{1}{2} \int_{\mathbb{R}^{3}} \epsilon(y) \nabla_{y} \psi(y) \cdot \nabla_{y} \psi(y) d y .
$$

It is a strictly positive number when $\rho \neq 0$.

The motivation of this definition is the thought experiment of bringing in the charges $\rho, q$ from infinity so at time $t \in[0,1]$ the charge distributions is $t \rho, t q$. At time $t$ the electrostatic potential energy is $-t \psi$ so the work performed in the interval $[t, t+d t]$ is equal to

$$
-\int_{\mathbb{R}^{3}}(t \psi)((\rho+q) d t) d y=-t d t \int_{\mathbb{R}^{3}} \psi(\rho+q) d y .
$$

Integrating from $t=0$ to $t=1$ yields the value of the definition.

\section{The DYNAmic EQUATions}

The potential energy $J$ of Definition 3.4 depends on the position of $K$ parametrized by $\operatorname{Euc}(3)$. The dynamics for the motion on $\operatorname{Euc}(3)$ is determined by the principle of stationary action,

$$
0=\delta \int_{t_{1}}^{t_{2}}(\text { Kinetic energy }- \text { Potential energy }) d t .
$$

This is a special case of the following. Suppose that $(\mathcal{R}, g)$ is an $n$-dimensional Riemannian manifold. Suppose that $J \in C^{1}(\mathcal{R} ; \mathbb{R})$. A motion $\left.\zeta \in C^{2}\left(\left[t_{1}, t_{2}\right] ; \mathcal{R}\right)\right)$ must satisfy

$$
0=\delta \int_{t_{1}}^{t_{2}} \frac{1}{2} g\left(\zeta^{\prime}(t), \zeta^{\prime}(t)\right)-J(\zeta(t)) d t
$$

among competing motions that have the same values as $\zeta$ at $t=t_{1}$ and $t=t_{2}$. In our example, $n=6=\operatorname{dim}(\operatorname{Euc}(3))$. The next result recalls familiar facts.

Proposition 4.1. i. In local coordinates $\zeta_{\alpha}$ on $\mathcal{R}$ the dynamics is defined by the action principal

$$
\delta \int_{t_{1}}^{t_{2}}\left(\frac{1}{2} \sum_{\alpha, \beta=1}^{n} g_{\alpha, \beta}\left(\zeta_{1}(t), \ldots, \zeta_{n}(t)\right) \frac{d \zeta_{\alpha}(t)}{d t} \frac{d \zeta_{\beta}(t)}{d t}-J(\zeta(t))\right) d t=0,
$$

with $g_{\alpha, \beta}=g_{\beta, \alpha}$ strictly positive definite. 
ii. To be a solution of the equations of motion is equivalent to the Euler-Lagrange system of ordinary differential equations in local coordinates,

$$
\frac{d}{d t} \sum_{\beta} g_{\alpha, \beta}(\zeta(t)) \frac{d}{d t} \zeta_{\beta}(t)=-\frac{\partial J(\zeta(t))}{\partial \zeta_{\alpha}}, \quad \text { for all } \alpha .
$$

iii. A point $\underline{\zeta}$ is an equilibrium if and only if it is a critical point of $J$.

iv. The stability and instability criteria of Theorems 1.10 and 1.11 apply in this more general situation.

The next result is needed in the proofs of the main Theorems 7.1 and 11.5.

Proposition 4.2. Suppose that $] a, b[\mapsto \zeta(t) \in \mathcal{R}$ is a geodesic of $(\mathcal{R}, g)$ parametrized proportional to arclength and $J \in C^{1}(\mathcal{R} ; \mathbb{R})$. If the values $\zeta(t)$ are all stationary points of $J$, then $\zeta$ satisfies the dynamic equation (4.3).

Proof. It is a classical result in Riemannian geometry that geodesics parametrized proportional to arclength satisfy the stationarity principal (4.2) with $J$ taken to be zero. Therefore in local coordinates

$$
\frac{d}{d t} \sum_{\beta} g_{\alpha, \beta}(\zeta(t)) \frac{d}{d t} \zeta_{\beta}(t)=0, \quad \text { for all } \alpha .
$$

On the other hand the fact that the values $\zeta(t)$ are stationary is equivalent to $\left.\nabla_{\zeta} J\right|_{\zeta(t)}=0$. This shows that the right hand side of (4.3) vanishes. Equation (4.3) is satisfied since both sides vanishes.

\section{ForCES AND TORQUES ON A CHARGED DIELECTRIC BODY}

The forces and torques on a dielectric body can be computed by adding forces and torques on particles or by the principle of virtual work. For completeness we verify that the two methods yield the same answer. The more elementary is to compute the forces on the charges $q$ and dipole charges div $p$. Summing yields

$$
\text { Total Force }=\int_{\mathbb{R}^{3}} E(y)(q(y)+\operatorname{div} p(y)) d y .
$$

Treating the torques analogously yields

$$
\text { Total Torque }=\int_{\mathbb{R}^{3}} y \wedge E(y)(q(y)+\operatorname{div} p(y)) d y .
$$

In the equations (5.1) and (5.2), the electric field $E$ is defined by (3.1).

Proposition 5.1. i. If $\rho \in H^{-1 / 2}\left(\mathbb{R}^{3}\right) \cap \mathcal{E}^{\prime}\left(\mathbb{R}^{3}\right)$ (where $\mathcal{E}^{\prime}\left(\mathbb{R}^{3}\right)$ denotes the space of distributions with compact support), denote by $E_{\rho} \in \dot{H}^{1 / 2}\left(\mathbb{R}^{3}\right)$ the solution of $-\operatorname{div} E_{\rho}=\rho$, 
$\operatorname{curl} E_{\rho}=0$. Then

$$
\int_{\mathbb{R}^{3}} E_{\rho}(y) \rho(y) d y=0 .
$$

ii. If for $j=1,2, \rho_{j} \in H^{-1 / 2}\left(\mathbb{R}^{3}\right) \cap \mathcal{E}^{\prime}\left(\mathbb{R}^{3}\right)$ and $E_{\rho_{j}} \in \dot{H}^{1 / 2}\left(\mathbb{R}^{3}\right)$ are the solutions with sources $\rho_{j}$, then

$$
\int_{\mathbb{R}^{3}} E_{\rho_{2}}(y) \rho_{1}(y) d y=-\int_{\mathbb{R}^{3}} E_{\rho_{1}}(y) \rho_{2}(y) d y
$$

Remark 5.2. 1. The integrals represent the pairing $H^{1 / 2} \times H^{-1 / 2} \mapsto \mathbb{C}$ that is the unique extension of $C_{0}^{\infty}\left(\mathbb{R}^{3}\right) \times C_{0}^{\infty}\left(\mathbb{R}^{3}\right) \ni \phi, \psi \mapsto \int \phi \psi d y$.

2. The first assertion shows that a charge distribution cannot exert a net force on itself. If it did the center of mass would accelerate under its own force.

3. Instead of the minimal smoothness $\rho \in H^{-1 / 2}\left(\mathbb{R}^{3}\right) \cap \mathcal{E}^{\prime}\left(\mathbb{R}^{3}\right)$, the simpler hypothesis $\rho \in L^{2}\left(\mathbb{R}^{3}\right) \cap \mathcal{E}^{\prime}\left(\mathbb{R}^{3}\right)$ yields $E_{\rho} \in \dot{H}^{1}\left(\mathbb{R}^{3}\right)$ and Proposition 5.1 remains true.

Proof. i. By continuity and density it suffices to consider $\rho \in C_{0}^{\infty}\left(\mathbb{R}^{3}\right)$ in which case the pairing is an absolutely convergent integral

$$
-\int_{\mathbb{R}^{3}} \rho(x) \frac{x-y}{4 \pi|x-y|^{3}} \rho(y) d x d y .
$$

The integrand satisfies $F(x, y)=-F(y, x)$ implying that its integral vanishes.

ii. The field of $\rho_{1}+\rho_{2}$ is equal to $E_{\rho_{1}}+E_{\rho_{2}}$. Part i. shows that

$0=\int_{\mathbb{R}^{3}} E_{\rho_{1}}(y) \rho_{1}(y) d y=\int_{\mathbb{R}^{3}} E_{\rho_{2}}(y) \rho_{2}(y) d y=\int_{\mathbb{R}^{3}}\left(E_{\rho_{1}}(y)+E_{\rho_{2}}(y)\right)\left(\rho_{1}(y)+\rho_{2}(y)\right) d y$.

Write the last as a sum of four terms. Two of them vanish by the first two equalities. This proves ii.

5.1. Formulas for the force. Denote by $\rho(x, \cdot):=\rho(y-x)$ the external charge translated by $x$. Denote by $K(x):=x+K$ the dielectric body translated by $x$. Denote by $\psi(x, \cdot)$ the potential in the translated geometry. Denote by $J(x)$ the electrostatic potential energy. They are well defined for $x$ small. The principal of virtual work asserts that the component of the force on $K$ in the $x_{1}$ direction is equal to $-\partial J / \partial x_{1}$ evaluated at $x=0$. The force is equal to $-\nabla_{x} J$.

The potential $\psi(x, y) \in \dot{H}^{1}\left(\mathbb{R}_{y}^{3}\right)$ is the unique solution of

$$
-\operatorname{div}_{y}\left(\epsilon(y-x) \nabla_{y} \psi\right)=\rho(y)+q(y-x) \text { in } \mathbb{R}^{3} .
$$

To compute $\nabla J(x)$ we reverse roles. Instead of fixing $\rho$ and translating $K$ we fix $K$ and translate $\rho$ by $-x$. Define $\widetilde{\psi}(x, y)$ and $\widetilde{\rho}(x, y)$ by

$$
\widetilde{\psi}(x, y):=\psi(x, x+y), \quad \text { and } \quad \widetilde{\rho}(x, y):=\rho(x+y) .
$$


The function $\widetilde{\psi}(x, \cdot) \in \dot{H}^{1}\left(\mathbb{R}_{y}^{3}\right)$ is the solution of

$$
-\operatorname{div}_{y}\left(\epsilon(y) \nabla_{y} \widetilde{\psi}\right)=\widetilde{\rho}(x, y)+q(y) \text { in } \mathbb{R}^{3} .
$$

The dependence on $x$ appears only in the source term.

Definition 5.3. Define

$$
\mathcal{O}:=\left\{x \in \mathbb{R}^{3}: K(x) \cap \operatorname{supp} \rho=\varnothing\right\} .
$$

The assumption that $K \cap \operatorname{supp} \rho=\varnothing$ implies that the set $\mathcal{O}$ contains a neighborhood of the origin 0 .

Proposition 5.4. If $\rho \in L^{2}\left(\mathbb{R}^{3}\right) \cap \mathcal{E}^{\prime}\left(\mathbb{R}^{3}\right)$, the map $\mathcal{O} \ni x \mapsto \widetilde{\psi}(x, \cdot)$ is a $C^{1}$ function with values in $\dot{H}^{1}\left(\mathbb{R}^{3}\right)$. The derivative $\partial \widetilde{\psi} / \partial x_{k}$ is the unique $\dot{H}^{1}\left(\mathbb{R}^{3}\right)$ solution of

$$
-\operatorname{div}_{y}\left(\epsilon(y) \nabla_{y} \frac{\partial \widetilde{\psi}}{\partial x_{k}}\right)=\frac{\partial \widetilde{\rho}}{\partial x_{k}} \text { in } \mathbb{R}^{3} .
$$

The energy $J$ is also continuously differentiable with

$$
-\frac{\partial J(x)}{\partial x_{k}}=-\int_{\mathbb{R}^{3}} \frac{\partial \widetilde{\rho}(x, y)}{\partial x_{k}} \widetilde{\psi}(x, y) d y=\int_{\mathbb{R}^{3}} \widetilde{\rho}(x, y) \frac{\partial \psi(x, y)}{\partial y_{k}} d y .
$$

When $\rho \in H^{1}\left(\mathbb{R}^{3}\right) \cap \mathcal{E}^{\prime}\left(\mathbb{R}^{3}\right)$, these expressions are equal to the $k^{\text {th }}$ component of (5.1).

Proof. Since $\rho$ is in $L^{2}\left(\mathbb{R}^{3}\right)$ the right hand side of (5.5) is a continuously differentiable function of $x$ with values in $\dot{H}^{1}\left(\mathbb{R}^{3}\right)$. The differentiability of $\psi$ follows. Formula (5.6) follows upon differentiating (5.5) with respect to $x$.

By definition

$$
J(x)=\frac{1}{2} \int_{\mathbb{R}^{3}}(\widetilde{\rho}+q) \widetilde{\psi} d y .
$$

Differentiating (5.8) with respect to $x_{k}$ yields

$$
2 \frac{\partial J(x)}{\partial x_{k}}=\int_{\mathbb{R}^{3}}\left(\frac{\partial \widetilde{\rho}(x, y)}{\partial x_{k}} \widetilde{\psi}(x, y)+(\widetilde{\rho}+q) \frac{\partial \widetilde{\psi}(x, y)}{\partial x_{k}}\right) d y .
$$

Multiplying equation (5.5) by $\partial \widetilde{\psi} / \partial x_{k}$ and integrating by parts in $y$ yields

$$
\int_{\mathbb{R}^{3}} \epsilon \nabla_{y} \widetilde{\psi} \cdot \nabla_{y} \frac{\partial \widetilde{\psi}}{\partial x_{k}} d y=\int_{\mathbb{R}^{3}}(\widetilde{\rho}+q) \frac{\partial \widetilde{\psi}}{\partial x_{k}} d y .
$$

Multiplying (5.6) by $\widetilde{\psi}$ gives

$$
\int_{\mathbb{R}^{3}} \epsilon \nabla_{y} \widetilde{\psi} \cdot \nabla_{y} \frac{\partial \widetilde{\psi}}{\partial x_{k}} d y=\int_{\mathbb{R}^{3}} \frac{\partial \widetilde{\rho}}{\partial x_{k}} \widetilde{\psi} d y .
$$


Therefore the two summands on the right hand side of (5.9) are equal. This yields the first expression on the right of (5.7). Use $\partial \widetilde{\rho} / \partial x=\partial \widetilde{\rho} / \partial y$ then integrate by parts to find the second expression on the right of (5.7).

With $x$ fixed the $k^{\text {th }}$ component of the force is equal to $-\partial J / \partial x_{k}$. The second expression in (5.7) shows that is equal to the $k^{\text {th }}$ component of

$$
-\int_{\mathbb{R}^{3}} \rho(x, y) E(x, y) d y
$$

The electric field is equal to

$$
E:=-\nabla_{y} \psi(x, y)=\int_{\mathbb{R}^{3}} \frac{x-y}{4 \pi|x-y|^{3}}(\rho(x, y)+q(x, y)+\operatorname{div} p(x, y)) d y .
$$

Since $E \in L^{2}$ one has $\operatorname{div} \alpha E \in H^{-1}$. This makes the pairing with $\rho \in H^{1}$ continuous. The symmetry of Proposition 5.1 implies that

$$
\int_{\mathbb{R}^{3}} E(x, y)(\rho(x, y)+q(x, y)+\operatorname{div} p(x, y)) d y=0
$$

so

$$
-\int_{\mathbb{R}^{3}} \rho(x, y) E(x, y) d y=\int_{\mathbb{R}^{3}} E(x, y)(q(x, y)+\operatorname{div} p(x, y)) d y
$$

yielding formula (5.1).

5.2. Formulas for the torque. Consider rotations of the body $K$. Denote by

$$
R(t):=e^{t A} \quad \text { with } t \in \mathbb{R}, \quad A=-A^{T} .
$$

The potential $\psi \equiv \psi(y, R) \in \dot{H}^{1}\left(\mathbb{R}_{y}^{3}\right)$, is the unique solution of

$$
-\operatorname{div}_{y} \epsilon\left(R^{-1} y\right) \nabla_{y} \psi=\rho(y)+q\left(R^{-1} y\right) \text { in } \mathbb{R}^{3} .
$$

Rotate equation (5.10) to fix the body. Introduce $\widetilde{\psi}(y, R):=\psi(R y, R)$ and $\widetilde{\rho}(y, R):=$ $\rho(R y)$ to find

$$
-\operatorname{div}_{y} \epsilon(y) \nabla_{y} \widetilde{\psi}=\rho(R y)+q(y)=\widetilde{\rho}(y)+q(y) \text { in } \mathbb{R}^{3} .
$$

The energy in the electric field is

$$
\begin{aligned}
J(R) & =\frac{1}{2} \int_{\mathbb{R}^{3}} \epsilon\left(R^{-1} y\right) \nabla_{y} \psi(y, R) \cdot \nabla_{y} \psi(y, R) d y \\
& =\frac{1}{2} \int_{\mathbb{R}^{3}} \epsilon(y) \nabla_{y} \widetilde{\psi}(y, R) \cdot \nabla_{y} \widetilde{\psi}(y, R) d y .
\end{aligned}
$$

Denote by ' the derivative with respect to $t$ so

$$
J^{\prime}(t)=\frac{d}{d t}(J(R(t))) \quad \text { and } \quad \widetilde{\psi}^{\prime}(y, t)=\frac{d}{d t}(\widetilde{\psi}(y, R(t))) .
$$


Lemma 5.5. i. For $\rho \in L^{2}\left(\mathbb{R}^{3}\right) \cap \mathcal{E}^{\prime}\left(\mathbb{R}^{3}\right)$, the derivative $J^{\prime}(0)$ is given by

$$
J^{\prime}(0)=\int_{\mathbb{R}^{3}} A y \cdot \nabla_{y} \rho(y) \psi(y) d y=\int_{\mathbb{R}^{3}} A y \cdot E(y) \rho(y) d y .
$$

ii Furthermore, if there exists $\omega \in \mathbb{R}^{3}$ such that $A v=\omega \wedge v$, then

$$
J^{\prime}(0)=\omega \cdot \int_{\mathbb{R}^{3}} y \wedge E(y) \rho(y) d y .
$$

By definition the integral $\int y \wedge E(y) \rho(y) d y$ is called the torque exerted by the forces with density $E(y) \rho(y)$.

Proof. i. Differentiating (5.11) with respect to $t$ yields

$$
-\operatorname{div}_{y} \epsilon(y) \nabla_{y} \widetilde{\psi}^{\prime}=R^{\prime}(t) y \cdot \nabla \widetilde{\rho}(y) \text { in } \mathbb{R}^{3} .
$$

Multiplying (5.15) by $\widetilde{\psi}$ leads to

$$
\int_{\mathbb{R}^{3}} \epsilon(y) \nabla_{y} \widetilde{\psi}^{\prime} \cdot \nabla_{y} \widetilde{\psi} d y=\int_{\mathbb{R}^{3}} \widetilde{\psi} R^{\prime}(t) y \cdot \nabla \widetilde{\rho}(y) d y
$$

Differentiating (5.12) gives

$$
J^{\prime}(t)=\int_{\mathbb{R}^{3}} \epsilon(y) \nabla_{y} \widetilde{\psi}^{\prime} \cdot \nabla_{y} \widetilde{\psi} d y .
$$

Comparing the last two formulas yields the middle term of (5.13) since for $t=0, R(0)=I$, $R^{\prime}(0)=A$, and $\widetilde{\psi}=\psi$.

By rotation invariance of Lebesgue measure,

$$
\frac{d}{d t} \int_{\mathbb{R}^{3}} \rho(R(t) y) \psi(R(t) y) d y=0 .
$$

Differentiate under the integral and set $t=0$ to find

$$
\int_{\mathbb{R}^{3}} A y \cdot \nabla_{y} \rho(y) \psi(y) d y=-\int_{\mathbb{R}^{3}} A y \cdot \nabla_{y} \psi(y) \rho(y) d y=\int_{\mathbb{R}^{3}} A y \cdot E(y) \rho(y) d y
$$

proving the last expression in (5.13).

ii. If $A$ is of the form $y \mapsto \omega \wedge y$ with $\omega \in \mathbb{R}^{3}$, then

$$
A y \cdot E \rho=(\omega \wedge y) \cdot E \rho=\omega \cdot(y \wedge E \rho) .
$$

Inserting this in (5.13) yields Equation (5.14).

Formula (5.14) for the torque is not the same as (5.2). The proof of equality in Example 5.8 relies on Newton's third law that we discuss next.

It is physically obvious that a charge distribution cannot exert a torque on itself. Otherwise it would set itself into rotary motion, violating conservation of angular momentum. The next equivalent result asserts that the torque exerted by the distribution $\rho_{1}$ on the charges $\rho_{2}$ is equal to minus the torque exerted by $\rho_{2}$ on $\rho_{1}$. 
Proposition 5.6. Suppose that $\rho_{j} \in H^{-1 / 2}\left(\mathbb{R}^{3}\right) \cap \mathcal{E}^{\prime}\left(\mathbb{R}^{3}\right)$ for $j=1,2$ and $E_{\rho_{j}}$ are their fields. Then the torque of the forces with density $E_{\rho_{1}} \rho_{2}$ is equal to minus the torque of the forces with density $E_{\rho_{2}} \rho_{1}$.

Proof. The torque of the forces with density $E_{\rho_{1}} \rho_{2}$ is equal to

$$
c \int_{\mathbb{R}^{3}} \int_{\mathbb{R}^{3}} y \wedge \frac{\rho_{2}(y) \rho_{1}(x)(y-x)}{|y-x|^{3}} d x d y .
$$

The integral sign is a shorthand for the pairing of $\rho_{2} \in H^{-1 / 2} \cap \mathcal{E}^{\prime}$ and $y \wedge E_{\rho_{1}} \in H_{\text {loc }}^{1 / 2}$, and

$$
E_{\rho_{1}}=c \int_{\mathbb{R}^{3}} \frac{\rho_{1}(x)(y-x)}{|y-x|^{3}} d y \in H^{1 / 2}\left(\mathbb{R}^{3}\right) .
$$

The torque with density $E_{\rho_{2}} \rho_{1}$ is

$$
c \int_{\mathbb{R}^{3}} \int_{\mathbb{R}^{3}} y \wedge \frac{\rho_{1}(y) \rho_{2}(x)(y-x)}{|y-x|^{3}} d x d y
$$

Changing the variable $y$ to $x$ and $x$ to $y$ shows this is equal to

$$
-c \int_{\mathbb{R}^{3}} \int_{\mathbb{R}^{3}} x \wedge \frac{\rho_{1}(x) \rho_{2}(y)(y-x)}{|y-x|^{3}} d x d y
$$

The sum of the two is equal to

$$
c \int_{\mathbb{R}^{3}} \int_{\mathbb{R}^{3}}(y-x) \wedge \frac{\rho_{2}(y) \rho_{1}(x)(y-x)}{|y-x|^{3}} d x d y .
$$

The sum vanishes because $(y-x) \wedge(y-x)=0$.

Example 5.7. If $\rho \in H^{-1 / 2}\left(\mathbb{R}^{3}\right) \cap \mathcal{E}^{\prime}\left(\mathbb{R}^{3}\right)$ and $E_{\rho}$ is the corresponding electric field then $E_{\rho} \in \dot{H}^{1 / 2}\left(\mathbb{R}^{3}\right)$ and the torque of the force field $\rho E_{\rho}$ makes sense and vanishes. This follows from applying the proposition with $\rho_{1}=\rho_{2}$. This proves that the torque exerted by a charge distribution on itself vanishes.

Example 5.8. To prove formula (5.2) reason as follows. The field of the dielectric in the presence of the external charges $\rho$ and the fixed charges $q$ on $K$ is the electric field of the compactly supported charge distribution $\rho+q+\operatorname{div} p$. Proposition 5.6 implies that

$$
\int_{\mathbb{R}^{3}} y \wedge E(\rho+q+\operatorname{div} p) d y=0 .
$$

This together with the formula from part ii of Lemma 5.5 yield formula (5.2).

Remark 5.9. i. A necessary condition for an equilibrium is that the energy $J$ is stationary under infinitesimal rotations, equivalently $J^{\prime}(0)=0$ for all antisymmetric $A$.

ii. The condition $J^{\prime}(0)=0$ for all anti-symmetric matrix $A$ holds if and only if $M=$ $\int_{\mathbb{R}^{3}} y \otimes \nabla_{y} \rho(y) \psi(y) d y$ is a symmetric matrix, where $y \otimes \nabla_{y} \rho(y)$ is the linear transformation $w \mapsto y\left(w \cdot \nabla_{y} \rho(y)\right)$. 


\section{The laplacian of the potential energy of a Dielectric}

This section first recalls Duffin's Theorem that the potential energy $J(x)$, as a function of the translation vector $x \in \mathbb{R}^{3}$, satisfies $\Delta J \leq 0$. Our first main result is a proof that at points $\underline{x}$ where $\Delta J(\underline{x})=0$, the electric field must be constant on a neighborhood of the dielectric.

Lemma 6.1. If $\rho \in H^{1}(\Omega) \cap \mathcal{E}^{\prime}(\Omega)$, then for all $x \in \mathcal{O}$,

$$
\Delta J(x)=\sum_{k=1}^{3} \int_{\mathbb{R}^{3}} \epsilon(y) \nabla_{y} \frac{\partial \widetilde{\psi}}{\partial x_{k}} \cdot \nabla_{y} \frac{\partial \widetilde{\psi}}{\partial x_{k}} d y-\int_{\mathbb{R}^{3}} \widetilde{\rho}^{2} d y .
$$

Proof. Differentiate (5.7) with respect to $x_{k}$ and use $\nabla_{x} \widetilde{\rho}=\nabla_{y} \widetilde{\rho}$ to find

$$
\frac{\partial^{2} J}{\partial x_{k}^{2}}=\int_{\mathbb{R}^{3}}\left(\frac{\partial \widetilde{\psi}}{\partial x_{k}} \frac{\partial \widetilde{\rho}}{\partial x_{k}}+\widetilde{\psi} \frac{\partial^{2} \widetilde{\rho}}{\partial y_{k}^{2}}\right) d y
$$

Multiplying (5.6) by $\partial \widetilde{\psi} / \partial x_{k}$ yields

$$
\int_{\mathbb{R}^{3}} \epsilon(y) \nabla_{y} \frac{\partial \widetilde{\psi}}{\partial x_{k}} \cdot \nabla_{y} \frac{\partial \widetilde{\psi}}{\partial x_{k}} d y=\int_{\mathbb{R}^{3}} \frac{\partial \widetilde{\psi}}{\partial x_{k}} \frac{\partial \widetilde{\rho}}{\partial x_{k}} d y=\int_{\mathbb{R}^{3}} \frac{\partial \widetilde{\psi}}{\partial x_{k}} \frac{\partial \widetilde{\rho}}{\partial y_{k}} d y
$$

Since $\widetilde{\rho}$ is compactly supported in $\mathbb{R}^{3}$, two integrations by parts yield

$$
\int_{\mathbb{R}^{3}} \widetilde{\psi} \frac{\partial^{2} \widetilde{\rho}}{\partial y_{k}^{2}} d y=-\int_{\mathbb{R}^{3}} \frac{\partial \widetilde{\psi}}{\partial y_{k}} \frac{\partial \widetilde{\rho}}{\partial y_{k}} d y=\int_{\mathbb{R}^{3}} \widetilde{\rho} \frac{\partial^{2} \widetilde{\psi}}{\partial y_{k}^{2}} d y
$$

Therefore

$$
\frac{\partial^{2} J}{\partial x_{k}^{2}}=\int_{\mathbb{R}^{3}} \epsilon(y) \nabla_{y} \frac{\partial \widetilde{\psi}}{\partial x_{k}} \cdot \nabla_{y} \frac{\partial \widetilde{\psi}}{\partial x_{k}} d y+\int_{\mathbb{R}^{3}} \widetilde{\rho} \frac{\partial^{2} \widetilde{\psi}}{\partial y_{k}^{2}} d y
$$

Summing (6.3) with respect to $k$ yields

$$
\Delta_{x} J(x)=\sum_{k=1}^{3} \int_{\mathbb{R}^{3}} \epsilon(y) \nabla_{y} \frac{\partial \widetilde{\psi}}{\partial x_{k}} \cdot \nabla_{y} \frac{\partial \widetilde{\psi}}{\partial x_{k}} d y+\int_{\mathbb{R}^{3}} \widetilde{\rho} \Delta_{y} \widetilde{\psi} d y .
$$

Since the support of $\widetilde{\rho}$ is disjoint from the dielectric body $K$ and the fixed charges $q$ are attached to $K$, Poisson's equation (5.5), restricted to the support of $\widetilde{\rho}$ is

$$
-\Delta_{y} \widetilde{\psi}=\widetilde{\rho} \quad \text { in } \mathbb{R}^{3} \backslash K . \quad \text { so, } \quad \int_{\mathbb{R}^{3}} \widetilde{\rho} \Delta_{y} \widetilde{\psi} d y=-\int_{\mathbb{R}^{3}} \widetilde{\rho}^{2} d y .
$$

This completes the proof of Lemma 6.1.

Part $\mathbf{i}$ of the next Theorem is due to Duffin [4] (see also [3], [5]).

Theorem 6.2. i. Suppose $\rho \in H^{1}\left(\mathbb{R}^{3}\right) \cap \mathcal{E}^{\prime}(\Omega)$, then

$$
\Delta J(x) \leq 0 \quad \text { on } \quad \mathcal{O} .
$$


ii. Furthermore if $\rho$ is not identically equal to zero, $\epsilon$ is strictly larger than $I$ on an open set $K_{0}$, and $\underline{x} \in \mathcal{O}$ with $\Delta J(\underline{x})=0$, then there is a neighborhood of $K$ and a vector $v \in \mathbb{R}^{3}$ so that the external field $E_{\text {ext }}$ from (3.2) is equal to $v$ on the neighborhood. In addition $J$ is an affine function on a neighborhood of $\underline{x}$.

The proof of $\mathbf{i}$ uses the complementary minimization principal from the next lemma.

Lemma 6.3. If $\zeta \in \dot{H}^{1}\left(\mathbb{R}^{3}\right)$ satisfies $-\operatorname{div} \epsilon \nabla \zeta=f$ then

$$
\int_{\mathbb{R}^{3}} \epsilon \nabla \zeta \cdot \nabla \zeta d y=\min _{\tau \in \mathcal{T}} \int_{\mathbb{R}^{3}} \epsilon^{-1} \tau \cdot \tau d y
$$

where

$$
\mathcal{T}=\left\{\tau \in L^{2}\left(\mathbb{R}^{3} ; \mathbb{R}^{3}\right):-\operatorname{div}_{y} \tau=f \quad \text { on } \quad \mathbb{R}^{3}\right\}
$$

The minimum is uniquely attained at $\tau=\epsilon \nabla \zeta \in \mathcal{T}$.

Proof of Lemma 6.3. The test function $\epsilon \nabla \zeta$ achieves equality in (6.6) so the minimum is smaller or equal to the left hand side. The existence of a minimizer is easy. If $\underline{\tau}$ is such a minimizer, the Euler-Lagrange equation asserts that

$$
\int_{\mathbb{R}^{3}} \epsilon^{-1} \underline{\tau} \cdot w d y=0 \text { for all } w \in L^{2} \quad \text { with } \quad \operatorname{div} w=0 .
$$

This is equivalent to $\operatorname{curl} \epsilon^{-1} \tau=0$ in the sense of distributions. In this case, explicit solution in Fourier shows that there is a unique $\chi \in \dot{H}^{1}\left(\mathbb{R}^{3}\right)$ with $\nabla \chi=\epsilon^{-1} \underline{\tau}$. Then $-\operatorname{div} \epsilon \nabla \chi=f$ so $\chi=\zeta$ by uniqueness. This proves that the minimum is achieved at and only at $\underline{\tau}=\epsilon \nabla \zeta$.

Proof of Theorem 6.2. Step I. Proof of (6.5) The complementary energy minimization principle yields

$$
\int_{\mathbb{R}^{3}} \epsilon \nabla_{y} \frac{\partial \widetilde{\psi}}{\partial x_{k}} \cdot \nabla_{y} \frac{\partial \widetilde{\psi}}{\partial x_{k}} d y=\min _{\tau \in \mathcal{T}} \int_{\mathbb{R}^{3}} \epsilon^{-1} \tau \cdot \tau d y,
$$

where

$$
\mathcal{T}=\left\{\tau \in L^{2}\left(\mathbb{R}^{3} ; \mathbb{R}^{3}\right):-\operatorname{div}_{y} \tau=\frac{\partial \widetilde{\rho}}{\partial x_{k}} \text { on } \mathbb{R}^{3}\right\} .
$$

Fix $k$. Take a test field $\tau=\nabla_{y} w_{k}$ with $w_{k}$ equal to the solution of

$$
-\Delta_{y} w_{k}=\frac{\partial \widetilde{\rho}}{\partial x_{k}} \quad \text { on } \quad \mathbb{R}^{3} .
$$

This yields the first of the following inequalities.

$$
\int_{\mathbb{R}^{3}} \epsilon \nabla_{y} \frac{\partial \widetilde{\psi}}{\partial x_{k}} \cdot \nabla_{y} \frac{\partial \widetilde{\psi}}{\partial x_{k}} d y \leq \int_{\mathbb{R}^{3}} \epsilon^{-1} \nabla_{y} w_{k} \cdot \nabla_{y} w_{k} d y \leq \int_{\mathbb{R}^{3}}\left|\nabla_{y} w_{k}\right|^{2} d y .
$$


The second holds because $\epsilon \geq I$. However,

$$
w_{k}=(-\Delta)^{-1} \frac{\partial \widetilde{\rho}}{\partial y_{k}} \quad \text { so, } \quad \sum_{k} \frac{\partial w_{k}}{\partial y_{k}}=(-\Delta)^{-1} \Delta \widetilde{\rho}=-\widetilde{\rho} .
$$

Therefore

$$
\begin{aligned}
\sum_{k} \int_{\mathbb{R}^{3}}\left|\nabla_{y} w_{k}\right|^{2} d y & =\sum_{k} \int_{\mathbb{R}^{3}} w_{k}\left(-\Delta w_{k}\right) d y=\sum_{k} \int_{\mathbb{R}^{3}} w_{k} \frac{\partial \widetilde{\rho}}{\partial y_{k}} d y \\
& =-\int_{\mathbb{R}^{3}} \sum_{k} \frac{\partial w_{k}}{\partial y_{k}} \widetilde{\rho} d y=\int_{\mathbb{R}^{3}}|\widetilde{\rho}|^{2} d y .
\end{aligned}
$$

Combined with (6.7), this yields

$$
\sum_{k} \int_{\mathbb{R}^{3}} \epsilon \nabla_{y} \frac{\partial \widetilde{\psi}}{\partial x_{k}} \cdot \nabla_{y} \frac{\partial \widetilde{\psi}}{\partial x_{k}} d y \leq \int_{\mathbb{R}^{3}}|\widetilde{\rho}|^{2} d y .
$$

The inequalities (6.8) and (6.1) imply (6.5).

Step II. When $\Delta J=0$ the external field is constant on a neighborhood of $K$. Consider a point $\bar{x}$ for which equality holds in (6.8), that is $\Delta_{x} J(\bar{x})=0$. Both inequalities in (6.7) must be equalities. When the first inequality in (6.7) is an equality, one has

$$
\nabla_{y} w_{k}(\bar{x}, \cdot)=\epsilon \nabla_{y} \frac{\partial \widetilde{\psi}(\bar{x}, \cdot)}{\partial x_{k}} \quad \text { in } \quad \mathbb{R}_{y}^{3}
$$

because there is a unique minimizer for the complementary minimum principal. Let $K_{0}:=\{x: \epsilon(x)>I\}$ a non empty open set by hypothesis. Equality in the second inequality in (6.7) implies that on $K_{0}$ where $\epsilon>I$,

$$
\left.\nabla_{y} w_{k}(\bar{x}, \cdot)\right|_{K_{0}}=0
$$

Since $K$ is connected, it belongs to a single connected component $\omega$ of $\mathbb{R}^{3} \backslash \operatorname{supp} \widetilde{\rho}(\bar{x}, \cdot)$. The function $w_{k}(\bar{x}, \cdot)$ is harmonic on $\mathbb{R}^{3} \backslash \operatorname{supp} \widetilde{\rho}(\bar{x}, \cdot) \supset \omega$. That harmonic function is

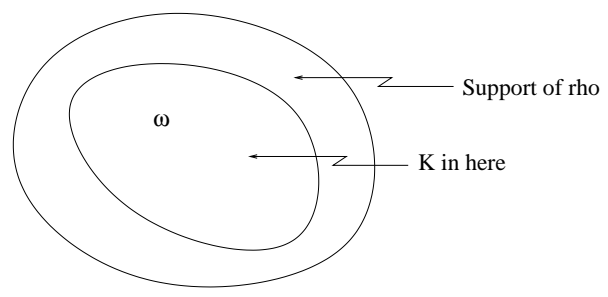

constant on $K_{0}$ so by analytic continuation, $\left.w_{k}(\bar{x}, \cdot)\right|_{\omega}$ is constant. Equation (6.9) then implies that $\partial \widetilde{\psi}(\bar{x}, \cdot) /\left.\partial x_{k}\right|_{\omega}$ is constant. There is a constant $v_{k}$ so that

$$
w_{k}(\bar{x}, y)=\frac{\partial \widetilde{\psi}(\bar{x}, y)}{\partial x_{k}}=v_{k}, \quad \text { for } \quad y \in \omega
$$


By definition both $\widetilde{\rho}$, and $w_{k}$ depend only on $x+y$. Therefore

$$
w_{k}(x, y)=v_{k} \quad \text { for } \quad x+y-\bar{x} \in \omega .
$$

Define the function $\widetilde{\phi}$ as the unique solution of

$$
-\Delta_{y} \widetilde{\phi}=\widetilde{\rho} \text { in } \mathbb{R}^{3}
$$

Compare to (6.12) to find

$$
w_{k}(x, y)=\frac{\partial \widetilde{\phi}}{\partial x_{k}}(x, y) .
$$

Therefore $\nabla_{x} \widetilde{\phi}(x, y)=\left(v_{1}, v_{2}, v_{3}\right)$ on a neighborhood of $K$.

The solution $\widetilde{\phi}(x, y)$ is a function of $(x+y)$, as is $\widetilde{\rho}$. Thus, there exist a vector $v \in \mathbb{R}^{3}$ and a constant $c \in \mathbb{R}$ such that, for $x, y$ on a neighborhood of $\bar{x} \times K$ in $\mathbb{R}_{x}^{3} \times \mathbb{R}_{y}^{3}$,

$$
\widetilde{\phi}(x, y)=v \cdot(x+y)+c .
$$

The external electric field is constant on a neighborhood of $K$.

Step III. When $\Delta J=0, J$ is affine. Since the gradient of $w_{k}$, vanishes wherever $\epsilon \neq I$, so $w_{k}(x, y)$ is also a solution of

$$
-\operatorname{div}_{y}\left(\epsilon(y) \nabla_{y} w_{k}\right)=-\Delta_{y} w_{k}=\frac{\partial \widetilde{\rho}}{\partial x_{k}} \text { in } \mathbb{R}_{y}^{3} .
$$

By uniqueness, for any $x$ sufficiently close to $\bar{x}$,

$$
w_{k}(x, y)=\frac{\partial \widetilde{\psi}}{\partial x_{k}}(x, y) \quad \text { in } \mathbb{R}_{y}^{3} .
$$

For $x$ in a small neighborhood of $\bar{x}, \widetilde{\psi}$ and $\widetilde{\phi}$ have the same gradient with respect to $x$ SO

Compute $J(x)$

$$
\widetilde{\psi}(x, y)=\widetilde{\phi}(x, y)+\phi_{0}(y)
$$

$$
2 J(x)=\int_{\mathbb{R}^{3}}(\widetilde{\rho}+q) \widetilde{\psi} d y=\int_{\mathbb{R}^{3}}\left(q \phi_{0}+\widetilde{\rho} \tilde{\phi}+q \widetilde{\phi}+\widetilde{\rho} \phi_{0}\right) d y .
$$

In the right hand side of (6.13) the first term is independent of $x$. The second term is independent of $x$ by translation invariance of Lebesgue measure. The third term is affine in $x$ since $\widetilde{\phi}=v \cdot(x+y)+c$ on the support of $q$. The fourth term is, by integration by parts,

$$
\int_{\mathbb{R}^{3}} \tilde{\rho} \phi_{0} d y=-\int_{\mathbb{R}^{3}} \Delta_{y} \widetilde{\phi} \phi_{0} d y=-\int_{\mathbb{R}^{3}} \widetilde{\phi} \Delta_{y} \phi_{0} d y=a v \cdot x+b, \quad a, b \in \mathbb{R}
$$

since $\widetilde{\phi}=v \cdot(x+y)+c$ in a neighborhood of $K$ and

$$
\Delta_{y} \phi_{0}=\Delta_{y} \tilde{\psi}-\Delta_{y} \tilde{\phi}=0 \quad \text { on } \quad \mathbb{R}^{3} \backslash K .
$$

Thus $J(x)$ is an affine function for $x$ close to $\bar{x}$. 
Remark 6.4. This result does not assume that $\underline{x}$ is an equilibrium. If $\underline{x}$ is an equilibrium, then $\nabla J(\underline{x})=0$. Then $J$ is affine with gradient vanishing at a point so $J$ is constant on a neighborhood of $\underline{x}$. This argument does not use the fact that the torques vanish at an equilibrium. If the torques do vanish, it does not imply the absence of torques after small rotation.

\section{INSTABILITY OF A DIELECTRIC BODY}

We now prove our first main instability result using part ii of Theorem 6.2.

Theorem 7.1. Suppose that $K$ a connected dielectric body with strictly positive polarizability on an open set. If $K(\underline{x})$ is an equilibrium position in the field of external charges $\rho \in H^{1}\left(\mathbb{R}^{3}\right) \cap \mathcal{E}^{\prime}\left(\mathbb{R}^{3}\right)$ with $K(\underline{x}) \cap \operatorname{supp} \rho=\varnothing$, then the equilibrium is unstable.

Remark 7.2. The renormalization technique of $\S 10$ shows that the conclusion is true for $\rho \in \mathcal{E}^{\prime}\left(\mathbb{R}^{3}\right)$ with $K(\underline{x}) \cap \operatorname{supp} \rho=\varnothing$, see Remark 11.6.

Proof. Dichotomy I. When $\Delta J(\underline{x})<0$ the hessian with respect to $x$ of the potential energy has a negative eigenvalue. The minimum eigenvalue of the hessian on the configuration space $\operatorname{Euc}(3)$ is smaller so is also negative. Theorem 1.11 with Proposition 4.1.iv imply instability.

Dichotomy II. In the opposite case $\Delta J(\underline{x})=0$, denote by $\underline{\rho}, \underline{q}, \underline{p}, \underline{E}$ values of the external charge, fixed charges, polarization, and electric field for the dielectric $K(\underline{x})$. Theorem 6.2 implies that the electric field $E_{\underline{\rho}}$ is equal to a constant vector $v$ on a neighborhood of $K(\underline{x})$.

Choose $0<\delta<\operatorname{dist}(K(\underline{x}), \operatorname{supp} \underline{\rho})$ so that $E_{\rho}$ is constant on $\left\{x \in \mathbb{R}^{3}: \operatorname{dist}(x, K(\underline{x}))<\right.$ $\delta$. We show that for $h \in \mathbb{R}^{3}$ with $\|\bar{h}\|<\delta$, the position $K(\underline{x}+h)$ is also an equilibrium of the dielectric. This is equivalent to showing that the position $K(\underline{x})$ with its fixed charges $\underline{q}$ is an equilibrium in the presence of the external charge distribution $\rho(\cdot-h)$. To do that we verify that the forces and torques vanishing for the charge distribution $\rho(\cdot-h)$.

Fix $h$ and consider the two external charge distributions $\rho$ and $\widetilde{\rho}:=\rho(\cdot-h)$ with fixed dielectric $K(\underline{x})$ and fixed charges $\underline{q}$. Since the two external charge distributions generate electric fields that are identical on a neighborhood of $K(\underline{x})$, part ii of Remark 3.3 implies that the polarizations $p$ and $\widetilde{p}$ are identical. Since the electric fields are equal to $v$ on a neighborhood of $K(\underline{x})$ formulas (5.1) and (5.2) show that

$$
\text { Force }=v \int q+\operatorname{div} p d x, \quad \text { Torque }=\int y \wedge v(q+\operatorname{div} p) d x .
$$

The values of $q, p$ are equal to $\underline{q}, \underline{p}$ are independent of $h$ so the force and torque are also independent of $h$. Since they vanish for the external charge distribution $\rho$ they also vanish for $\widetilde{\rho}$. Therefore, all small translates of $K$ are equilibria. 
Part ii of Proposition 2.4 implies that $t \mapsto M(v t)$ is a geodesic of Euc(3) parametrized proportional to arclength. We have just shown that each point on this trajectory is an equilibrium, that is a stationary point of $J$. Proposition 4.2 implies that the dynamic equations (4.3) are satisfied.

The motions $M(t v) K(\underline{x})$ with $v$ arbitrarily small start as close to the equilibrium as one likes in the sense of Cauchy data. Indeed the positions coincide and the velocity of the equilibrium vanishes while the velocity of $M(t v) K(\underline{x})$ is arbitrarily small. The motion moves steadily away to a distance $\sim \delta$ proving instability.

\section{Field AND ENERGy OF A CHARGED PERFECT CONDUCTOR}

This section presents the analysis for the case of a perfect conductor body $K$.

8.1. Field and energy of a conductor. Suppose that $\rho \in H^{-1}\left(\mathbb{R}^{3}\right) \cap \mathcal{E}^{\prime}\left(\mathbb{R}^{3}\right)$. with $K \cap \operatorname{supp} \rho=\varnothing$. Suppose that $K$ has net charge equal to $\sigma$, possibly zero, that is free to move on the perfect conductor.

In this section, the potential is now the unique $\psi \in \dot{H}^{1}\left(\mathbb{R}^{3}\right)$ satisfying

$$
-\Delta \psi=\rho,\left.\quad \psi\right|_{K}=\text { constant }, \quad \sigma=-\int_{\partial \Omega} \mathbf{n} \cdot \nabla \psi d S .
$$

The electric field $E$ vanishes on $K$ and is equal to $\nabla \psi$ on $\Omega$. Therefore $\operatorname{div} E=-\rho+$ $(E \cdot \mathbf{n}) d S$ with the values of $E$ computed from $\Omega$. There a surface charge density equal to $-\mathbf{n} \cdot \nabla \psi d S$. The solution is $C^{\infty}(\bar{\Omega} \backslash \operatorname{supp} \rho)$ and in the flux integral, the trace of $\nabla \psi$ is taken from the interior of $\Omega$. If $K$ were not connected the condition on $\left.\psi\right|_{K}$ would be replaced by constant values on each connected component of $K$.

Definition 8.1. Define $v(x)$ to be the capacitory potential of $K$ that is the solution

$$
\Delta v=0 \text { on } \Omega, \quad v=1 \text { on } K, \quad v=O(1 /|x|) \text { as }|x| \rightarrow \infty .
$$

The capacity $C a p(K)$ is defined by

$$
\operatorname{Cap}(K)=-\int_{\partial \Omega} \frac{\partial v}{\partial \nu} d \Sigma
$$

The external field $E_{\text {ext }}$ and potential $\phi_{\text {ext }}$ are defined in (3.2). The potential $\psi$ is uniquely determined by the values of the external potential $\phi_{\text {ext }}$ on a neighborhood of $K$.

Proposition 8.2. Denote by $-\psi$ the electrostatic potential of the conductor $K$ with charge $\sigma$ and external source $\rho$. With $\phi_{\text {ext }}$ from (3.2), define $\zeta$ by $\left.\zeta\right|_{K}=-\left.\phi_{\text {ext }}\right|_{K}$, and $\left.\zeta\right|_{\Omega}$ equal to the solution of the Dirichlet problem

$$
\Delta \zeta=0 \quad \text { on } \quad \Omega,\left.\quad \zeta\right|_{\partial \Omega}=-\left.\phi_{\text {ext }}\right|_{\partial \Omega}, \quad \zeta=O(1 /|x|) \quad \text { as } \quad|x| \rightarrow \infty .
$$


Then,

$$
\psi=\phi_{\text {ext }}+\zeta+c v, \quad c=\frac{1}{\operatorname{Cap}(K)}\left(\sigma+\int_{\partial \Omega} \frac{\partial \phi_{\text {ext }}}{\partial \nu} d \Sigma\right)
$$

Proof. For any value of $c$ one has $\psi \in \dot{H}^{1}\left(\mathbb{R}^{3}\right), \Delta \psi=\rho$ on $\Omega$, and $\left.\psi\right|_{K}$ constant. The charge on $K$ is equal

$$
c \operatorname{Cap}(K)-\int_{\partial \Omega} \frac{\partial \phi_{\text {ext }}}{\partial \nu} d \Sigma .
$$

Setting this equal to $\sigma$ determines $c$ and yields (8.2).

When $\rho \in H^{-1}\left(\mathbb{R}^{3}\right) \cap \mathcal{E}^{\prime}\left(\mathbb{R}^{3}\right)$ and $K$ is connected, the solution $\psi$ is characterized as minimizing

$$
\int_{\mathbb{R}^{3}}\left(\frac{1}{2}|\nabla \psi|^{2}+\rho \psi\right) d y-\frac{\sigma}{|\partial K|} \int_{\partial K} \psi d \Sigma
$$

over the set of $\psi \in \dot{H}^{1}\left(\mathbb{R}^{3}\right)$ whose restriction to $K$ is constant. In the case of several conductors $K_{j}$ with charges $\sigma_{j}$ the set of candidate functions would be the elements of $\psi \in \dot{H}^{1}\left(\mathbb{R}^{3}\right)$ with $\left.\psi\right|_{K_{j}}$ constant for each $j$. The boundary term in the minimization would be replaced by

$$
\sum_{j} \frac{\sigma_{j}}{\left|\partial K_{j}\right|} \int_{\partial K_{j}} \psi d \Sigma .
$$

Definition 8.3. For $\rho \in \mathcal{E}^{\prime}(\Omega) \cap \dot{H}^{-1}\left(\mathbb{R}^{3}\right)$ with $\operatorname{supp} \rho \cap K=\varnothing$, the energy in the electric field of the equilibrium charge distribution on the perfect conductor $K$ carrying charge $\sigma$ is defined as

$$
J=\frac{1}{2} \int_{\mathbb{R}^{3}}|\nabla \psi|^{2} d y=\frac{1}{2} \int_{\Omega}|\nabla \psi|^{2} d y=-\frac{1}{2} \int_{\Omega} \rho \psi d y-\left.\frac{1}{2} \psi\right|_{K} \sigma .
$$

It is a strictly positive number when $\rho \neq 0$.

The physical motivation of this definition is to compute the work performed in assembling the charge distributions $t \rho, t \sigma$ for $0 \leq t \leq 1$. The work in the interval $d t$ carries charge $\rho d t$ and $\sigma d t$ from infinity in the potential $-t \psi$ for a net work equal to

$$
-\int\left(t \psi \rho d t+\left.t \psi\right|_{K} \sigma d t\right) d y \text {. }
$$

Integrating $\int_{0}^{1} d t$ yields the formula on the right of (8.3). The energy is exactly equal to the energy $\int|E|^{2} d y / 2$ in the electric field. In contrast, the total energy for a dielectric is larger than $\int|E|^{2} d x$.

Lemma 8.4. Suppose that $0 \neq \rho \in \dot{H}^{-1}\left(\mathbb{R}^{3}\right) \cap \mathcal{E}^{\prime}(\Omega)$. Then, $J$ is equal to

$$
J=\frac{1}{2} \int_{\mathbb{R}^{3}} \int_{\mathbb{R}^{3}} \rho(y) \frac{1}{4 \pi|x-y|} \rho(x) d x d y+\int_{\partial \Omega} \frac{\partial \psi(y)}{\partial \nu} \phi_{e x t}(y) d S(y)+\left.\psi\right|_{\partial \Omega} \sigma
$$


Proof. Begin with the identities

$$
\begin{aligned}
2 J & =-\int_{\mathbb{R}^{3}} \psi \Delta \psi d y=\int_{\mathbb{R}^{3}} \psi(y)\left(\rho(y) d y+\frac{\partial \psi(y)}{\partial \nu} d S(y)\right) \\
& =\int_{\mathbb{R}^{3}} \int_{\mathbb{R}^{3}}\left(\rho(y) d y+\frac{\partial \psi(y)}{\partial \nu} d S(y)\right) \frac{1}{4 \pi|x-y|}\left(\rho(x) d x+\frac{\partial \psi(x)}{\partial \nu} d S(x)\right),
\end{aligned}
$$

where $d S$ is the surface measure on $\partial \Omega$. Expanding the last expression in (8.5) yields

$$
\begin{aligned}
\int_{\mathbb{R}^{3}} \int_{\mathbb{R}^{3}} \rho(y) & \frac{1}{4 \pi|x-y|} \rho(x) d x d y+2 \int_{\mathbb{R}^{3}} \int_{\mathbb{R}^{3}} \frac{\partial \psi(y)}{\partial \nu} \frac{1}{4 \pi|x-y|} \rho(x) d x d S(y) \\
& +\int_{\partial \Omega} \int_{\partial \Omega} \frac{\partial \psi(y)}{\partial \nu} \frac{1}{4 \pi|x-y|} \frac{\partial \psi(x)}{\partial \nu} d S(y) d S(x) .
\end{aligned}
$$

In the middle term, integrating $d x$ first yields

$$
2 \int_{\mathbb{R}^{3}} \int_{\mathbb{R}^{3}} \frac{\partial \psi(y)}{\partial \nu} \frac{1}{4 \pi|x-y|} \rho(x) d x d S(y)=2 \int_{\partial \Omega} \frac{\partial \psi(y)}{\partial \nu} \phi_{\text {ext }}(y) d S(y) .
$$

For the last term in (8.6) use

$$
\int_{\mathbb{R}^{3}} \frac{1}{4 \pi|x-y|}\left(\rho(y) d y+\frac{\partial \psi(y)}{\partial \nu} d S(y)\right)=\psi(x),
$$

to find

$$
\int_{\partial \Omega} \frac{1}{4 \pi|x-y|} \frac{\partial \psi(y)}{\partial \nu} d S(y)=\psi(x)-\int_{\mathbb{R}^{3}} \frac{1}{4 \pi|x-y|} \rho(y) d y=\psi(x)-\phi_{\text {ext }}(x) .
$$

Therefore

$$
\int_{\partial \Omega} \int_{\partial \Omega} \frac{\partial \psi(y)}{\partial \nu} \frac{1}{4 \pi|x-y|} \frac{\partial \psi(x)}{\partial \nu} d S(y) d S(x)=\int_{\partial \Omega} \frac{\partial \psi(y)}{\partial \nu}\left(\psi(y)-\phi_{\text {ext }}(y)\right) d S(y) .
$$

Since $\psi$ is constant on the boundary one has

$$
\int_{\partial \Omega} \frac{\partial \psi(y)}{\partial \nu} \psi(y) d S(y)=\left.\psi\right|_{\partial \Omega} \int_{\partial \Omega} \frac{\partial \psi(y)}{\partial \nu} d S(y)=\left.\psi\right|_{\partial \Omega} \sigma
$$

So

$$
\int_{\partial \Omega} \int_{\partial \Omega} \frac{\partial \psi(y)}{\partial \nu} \frac{1}{4 \pi|x-y|} \frac{\partial \psi(x)}{\partial \nu} d S(y) d S(x)=\left.\psi\right|_{\partial \Omega} \sigma-\int_{\partial \Omega} \frac{\partial \psi(y)}{\partial \nu} \phi_{\text {ext }}(y) d S(y)
$$

Combining (8.6), (8.7), and (8.8) completes the proof of (8.4).

\section{Perturbation theory for a Perfect CONductor}

The forces exerted on the body $K$ are given by the derivatives of the function $J$. To compute those it suffices to consider the variations of the potential $\psi(x, y)$, defined by (8.1), for the displaced bodies $K(x)=x+K$. The functions $\psi(x, y)$ for different values of 
$x$ are defined on different sets. To compute the derivatives, transform to a fixed reference domain. Define $\widetilde{\psi}$ and $\widetilde{\rho}(x, y)$ by

$$
\widetilde{\psi}(x, y):=\psi(x, x+y), \quad \text { and } \quad \widetilde{\rho}(x, y):=\rho(x+y) .
$$

The function $\widetilde{\psi}$ is characterized as the solution of the boundary value problem on the fixed domain $\Omega$,

$$
-\Delta_{y} \widetilde{\psi}=\widetilde{\rho} \quad \text { on } \Omega,\left.\quad \widetilde{\psi}\right|_{K}=\text { constant }, \quad \sigma=\int_{\partial \Omega} \frac{\partial \widetilde{\psi}}{\partial \nu} d S(y) .
$$

Proposition 9.1. i. $\widetilde{\psi}(x, y)$ is a smooth function on the set of $x, y$ so that $x \in \mathcal{O}$ and $x+y \notin \operatorname{supp} \rho$.

ii. If $\rho \in L^{2}\left(\mathbb{R}^{3}\right) \cap \mathcal{E}^{\prime}\left(\mathbb{R}^{3}\right)$ the derivative $\partial \widetilde{\psi} / \partial x_{k}$ is determined as the unique $\dot{H}^{1}\left(\mathbb{R}^{3}\right)$ solution to

$$
-\Delta_{y} \frac{\partial \widetilde{\psi}}{\partial x_{k}}=\frac{\partial \widetilde{\rho}}{\partial x_{k}} \text { on } \Omega,\left.\quad \frac{\partial \widetilde{\psi}}{\partial x_{k}}\right|_{K}=\text { constant }, \quad \int_{\partial \Omega} \frac{\partial}{\partial \nu}\left(\frac{\partial \widetilde{\psi}}{\partial x_{k}}\right) d S(y)=0 .
$$

Proof. i. Begin with the identity $\psi=\phi_{\text {ext }}+w$. Equivalently $\widetilde{\psi}(x, y)=\phi_{\text {ext }}(x+y)+\widetilde{w}(x, y)$ where $\widetilde{w}(x, y) \in \dot{H}^{1}\left(\mathbb{R}^{3}\right)$ is the unique solution of

(9.4) $-\Delta_{y} \widetilde{w}=0$ on $\Omega,\left.\quad \widetilde{w}\right|_{K}=-\phi_{e x t}(x+y)+$ constant, $\quad \int_{\partial \Omega} \frac{\partial \widetilde{w}(x, y)}{\partial \nu} d S(y)=\sigma$.

The source term $\phi_{\text {ext }}(x+y)$ is a smooth function of $x$ on a neighborhood of $K$ for all $x \in \mathcal{O}$. The smoothness of $\widetilde{w}$ and therefore of $\widetilde{\psi}$ follows.

ii. For $x \in \mathcal{O}$ consider equation (9.2). Since $\rho \in L^{2}\left(\mathbb{R}^{3}\right)$, the map $x \mapsto \widetilde{\rho}(x, \cdot)$ is a differentiable function of $x \in \mathcal{O}$ with values in $\dot{H}^{-1}\left(\mathbb{R}^{3}\right) \cap \mathcal{E}^{\prime}(\Omega)$. Therefore the map $x \mapsto \widetilde{\psi}(x, \cdot)$ is differentiable with values in $\dot{H}^{1}\left(\mathbb{R}^{3}\right)$. Differentiating (9.2) with respect to $x_{k}$ yields the boundary value problem (9.3).

Proposition 9.2. If $\rho \in L^{2}\left(\mathbb{R}^{3}\right) \cap \mathcal{E}^{\prime}\left(\mathbb{R}^{3}\right)$ and $x \in \mathcal{O}$ then

$$
\frac{\partial J(x)}{\partial x_{j}}=2 \int_{\Omega} \frac{\partial \widetilde{\rho}(x, y)}{\partial x_{j}} \widetilde{\psi}(x, y) d y .
$$

Equivalently,

$$
\frac{\partial J(x)}{\partial x_{j}}=\int_{\partial \Omega}\left|\nabla_{y} \widetilde{\psi}(x, y)\right|^{2} \nu \cdot e_{j} d y=-2 \int_{\Omega} \widetilde{\rho} \partial_{y_{1}} \widetilde{\psi} d y .
$$

Remark 9.3. i. Formula (9.5) is convenient for the computation of the second order derivatives of $J$.

ii. The first term on the right of (9.6), is in the standard form of a shape derivative satisfying the Hadamard structure theorem [7], namely written as a boundary integral depending linearly on the normal component of the displacement field. In this case the displacement field is a translation in the $e_{j}$ direction. 
Proof. Prove the case $j=1$. Start with

$$
J(x)=\int_{\Omega} \widetilde{\rho} \widetilde{\psi} d y+\left.\sigma \widetilde{\psi}\right|_{K} .
$$

The definition of $J$ yields

$$
J(x)=\int_{\Omega(x)}\left|\nabla_{y} \psi(x, y)\right|^{2} d y=\int_{\Omega}\left|\nabla_{y} \widetilde{\psi}(x, y)\right|^{2} d y .
$$

Differentiating (9.8) with respect to $x_{1}$ yields

$$
\frac{\partial J}{\partial x_{1}}=2 \int_{\Omega} \nabla_{y} \widetilde{\psi}(x, y) \cdot \partial_{x_{1}} \nabla_{y} \widetilde{\psi}(x, y) d y .
$$

Integration by parts in $y$ yields

$$
\frac{\partial J}{\partial x_{1}}=-2 \int_{\Omega} \Delta_{y} \widetilde{\psi}(x, y) \partial_{x_{1}} \widetilde{\psi}(x, y) d y+2 \int_{\partial \Omega} \frac{\partial \widetilde{\psi}}{\partial \nu} \partial_{x_{1}} \widetilde{\psi} d S(y)
$$

Since $\partial_{x_{1}} \widetilde{\psi}$ is constant on $K$ one finds

$$
\frac{\partial J}{\partial x_{1}}=-2 \int_{\Omega} \Delta_{y} \widetilde{\psi}(x, y) \partial_{x_{1}} \widetilde{\psi}(x, y) d y+\left.2 \sigma \frac{\partial \widetilde{\psi}}{\partial x_{1}}\right|_{K} .
$$

Using (9.2) yields

$$
\frac{\partial J}{\partial x_{1}}-\left.2 \sigma \frac{\partial \widetilde{\psi}}{\partial x_{1}}\right|_{K}=2 \int_{\Omega} \widetilde{\rho} \partial_{x_{1}} \widetilde{\psi}(x, y) d y=2 \int_{\Omega}\left(\partial_{x_{1}}(\widetilde{\rho} \widetilde{\psi}(x, y))-\left(\partial_{x_{1}} \widetilde{\rho}\right) \widetilde{\psi}\right) d y .
$$

Therefore (9.7) implies

$$
\begin{aligned}
\frac{\partial J}{\partial x_{1}}-\left.2 \sigma \frac{\partial \widetilde{\psi}}{\partial x_{1}}\right|_{K} & =2 \partial_{x_{1}} \int_{\Omega}(\widetilde{\rho} \widetilde{\psi}(x, y)) d y-2 \int_{\Omega}\left(\partial_{x_{1}} \widetilde{\rho}\right) \widetilde{\psi} d y \\
& =2 \frac{\partial\left(J-\left.\sigma \widetilde{\psi}\right|_{K}\right)}{\partial x_{1}}-2 \int_{\Omega}\left(\partial_{x_{1}} \widetilde{\rho}\right) \widetilde{\psi} d y
\end{aligned}
$$

From the definitions,

$$
\frac{\partial\left(\left.\widetilde{\psi}\right|_{K}\right)}{\partial x_{1}}=\left.\frac{\partial \widetilde{\psi}}{\partial x_{1}}\right|_{K}
$$

completing the proof of (9.5).

To obtain (9.6) from (9.5), first remark that $\partial_{x_{1}} \widetilde{\rho}=\partial_{y_{1}} \widetilde{\rho}$ and integrate by parts to get

$$
\frac{\partial J}{\partial x_{1}}=-2 \int_{\Omega} \widetilde{\rho} \partial_{y_{1}} \widetilde{\psi} d y+2 \int_{\partial \Omega} \tilde{\rho} \widetilde{\psi} \nu \cdot e_{1} d S(y)=-2 \int_{\Omega} \widetilde{\rho} \partial_{y_{1}} \widetilde{\psi} d y
$$

where the boundary integral vanishes since $\widetilde{\rho}=0$ on $K$. This proves the last expression in (9.6). Using (9.2) and another integration by parts yields

$$
\text { (9.10) } \frac{\partial J}{\partial x_{1}}=2 \int_{\Omega} \Delta_{y} \widetilde{\psi} \partial_{y_{1}} \widetilde{\psi} d y=-2 \int_{\Omega} \nabla_{y} \widetilde{\psi} \cdot \nabla_{y} \partial_{y_{1}} \widetilde{\psi} d y+2 \int_{\partial \Omega} \partial_{\nu} \widetilde{\psi} \partial_{y_{1}} \widetilde{\psi} d S(y) \text {. }
$$


Since $\widetilde{\psi}$ is constant on $\partial \Omega$, we have $\partial_{y_{1}} \widetilde{\psi}=\nu \cdot e_{1} \partial_{\nu} \widetilde{\psi}$ and $\left(\partial_{\nu} \widetilde{\psi}\right)^{2}=\left|\nabla_{y} \widetilde{\psi}\right|^{2}$ on $\partial \Omega$. Thus, (9.10) becomes

$$
\frac{\partial J}{\partial x_{1}}=-\int_{\Omega} \partial_{y_{1}}\left|\nabla_{y} \widetilde{\psi}\right|^{2} d y+2 \int_{\partial \Omega} \nu \cdot e_{1}\left|\nabla_{y} \widetilde{\psi}\right|^{2} d S(y) .
$$

The first integral on the right vanishes. This yields the middle expression in (9.6).

\section{Renormalized Potential ENERGy FOR $\rho$ With INFINITE SELF ENERGy}

The analysis up to here and the earlier work of Duffin is all in the context of charge distributions of finite energy that is $\rho \in \dot{H}^{-1}\left(\mathbb{R}^{3}\right)$. This has the unfortunate consequence of not permitting the classic example of an external point charge, This section explains how to treat external charges that are arbitrary compactly supported distributions $\rho \in \mathcal{E}^{\prime}\left(\mathbb{R}^{3}\right)$. A similar set of remarks works for the case of dielectrics too.

The first summand in (8.4) has the interpretation as the self energy of the charge distribution $\rho$. We are interested in forces on the body $K$. When $K$ moves the first summand does not change. The forces are determined by the changes in the second summand. Keeping only the two last terms in formula (8.4) for $J(x)$ yields the following definition of the renormalized energy $G(x)$.

Definition 10.1. For $x \in \mathcal{O}$ we introduce the renormalized potential energy

$$
G(x):=\int_{\partial \Omega(x)} \frac{\partial \psi(x, y)}{\partial \nu} \phi_{e x t}(y) d S(y)+\left.\psi\right|_{\partial \Omega(x)} \sigma,
$$

where $\psi$ is the solution to (8.1) and $\phi_{\text {ext }}=-\Delta^{-1} \rho$ is the solution of (3.2) in the sense of distributions. The potential $\phi_{\text {ext }}$ need not belong to $\dot{H}^{1}\left(\mathbb{R}^{3}\right)$.

Lemma 8.4 shows that for $\rho \in \dot{H}^{-1}\left(\mathbb{R}^{3}\right) \cap \mathcal{E}^{\prime}\left(\mathbb{R}^{3}\right), J(x)-G(x)$ is equal to the (finite) self energy of $\rho$ and is independent of $x \in \mathcal{O}$.

Proposition 10.2. i. If $\underline{x} \in \mathcal{O}$ then $\phi_{\text {ext }}=-\Delta^{-1} \rho$ is a smooth function in a neighborhood of $K(\underline{x})$. Its values in this neighborhood determine the value of $G(x) \in \mathbb{R}$ for $x$ on a neighborhood of $\underline{x}$.

ii. If $\omega \subset \Omega(\underline{x})$ is an open set and $0<r<\operatorname{dist}(\omega, K(\underline{x}))$ then the map $\rho \mapsto G$ is continuous from $\mathcal{E}^{\prime}(\omega) \rightarrow C^{\infty}\left(\bar{B}_{r}(\underline{x})\right)$.

iii. If $\rho \in \mathcal{E}^{\prime}\left(\mathbb{R}^{3}\right)$ and $\rho^{\epsilon} \in C_{0}^{\infty}\left(\mathbb{R}^{3}\right)$ is a smooth sequence converging to $\rho$ in the sense of distributions with $\operatorname{dist}\left\{\operatorname{supp} \rho, \mathbb{R}^{3} \backslash \operatorname{supp} \rho^{\epsilon}\right\} \rightarrow 0$, then the forces computed from the potential energy $J^{\epsilon}$, associated to $\rho^{\epsilon}$, are equal to those computed from $G^{\epsilon}$ and converge, as $\epsilon \rightarrow 0$, to the forces computed from $G$ for $\rho$. 
Proof. i. Since $\rho$ vanishes on a neighborhood of $K(x)$, one has

$$
\int_{\partial \Omega(x)} \frac{\partial \phi_{e x t}(y)}{\partial \nu} d S(y)=-\int_{K(x)} \Delta_{y} \phi_{e x t}(y) d y=0 .
$$

Decompose $\psi=\phi_{\text {ext }}+w$ where $w(x, y) \in \dot{H}^{1}\left(\mathbb{R}_{y}^{3}\right)$ is the unique solution of

$$
\begin{aligned}
-\Delta_{y} w & =0 \text { on } \Omega(x), \quad w=-\phi_{\text {ext }}+\text { constant on } K(x), \\
\sigma & =\int_{\partial \Omega(x)} \frac{\partial w(x, y)}{\partial \nu} d S(y), \quad w=O(1 /|x|) \quad|x| \rightarrow \infty .
\end{aligned}
$$

The function $w$ is determined by the values of $\phi_{\text {ext }}$ in a neighborhood of $K(x)$. Since $\psi=w+\phi_{\text {ext }}$, formula (10.1), shows that $G(x)$ also depends only on the values of $\phi_{\text {ext }}$ in a neighborhood of $K(x)$.

ii. For $x \in \bar{B}_{r}$ the support of $\rho$ is at finite distance from $K(x)$ so the map $\rho \mapsto \phi_{\text {ext }}$ is continuous with values in the functions smooth on an open set containing $\cup_{x \in \bar{B}_{r}} K(x)$. The formulas determining $\psi(x, y)$ and $G$ complete the proof.

iii. Immediate consequence of the continuity result of part ii.

\section{Renormalized COnductor energy $G$ SAtisfies $\Delta G \leq 0$}

Theorem 11.1. For any $\rho \in \mathcal{E}^{\prime}(\Omega)$, the function $G(x)$ satisfies

$$
\Delta_{x} G(x) \leq 0 \quad \text { for } \quad x \in \mathcal{O} .
$$

Furthermore if $K$ is connected, $\rho \in \mathcal{E}^{\prime}(\Omega)$ is not identically equal to zero, and $\underline{x} \in \mathcal{O}$ satisfies $\Delta_{x} G(\underline{x})=0$, then the electric field is constant on a neighborhood of $K$ and $G$ is an affine function on a neighborhood of $\underline{x}$.

Example 11.2. 1. If $\underline{x} \in \mathcal{O}$ is an equilibrium at which $\Delta_{x} G(\underline{x})=0$, then Theorem 11.1 states that $G$ is affine on a neighborhood of $\underline{x}$ and, because it is an equilibrium, $\nabla_{x} G(\underline{x})=0$, so $G$ is constant on a neighborhood of $\underline{x}$.

2. Consider spherically symmetric external charges $\rho$ surrounding a charge free region. The external potential $\phi_{\text {ext }}$ is constant in the charge free region. Since the energy $G$ is determined by the value of $\phi_{\text {ext }}$ on a neighborhood of $K$ it follows that for a perfect conductor in the charge free region, the potential $G(x)$ is independent of $x$. The renormalized energy is also independent of rotations.

3. Consider a perfectly conducting ellipsoid of revolution. Suppose that the external field is equal to $(1,0,0)$ on a neighborhood of the conductor. Then by translation invariance of the external field, the renormalized potential energy $G(x)$ is independent of $x$ for $x$ small. If the long axis of the ellipsoid is nearly parallel to $(1,0,0)$, then the induced surface charges will create a non trivial dipole moment and corresponding non vanishing torque. At an equilibrium position, the torque vanishes. In this case the torque also vanishes for 
$K(x)$ by translational symmetry of the field near $K$. Therefore $t \mapsto M(t v) K$ is a motion for $v \in \mathbb{R}^{3}$. Taking $|v| \ll 1$ proves instability.

The proof of Theorem 11.1 uses a formula for the the laplacian $\Delta J$ that requires additional regularity of $\rho$.

Lemma 11.3. If $\rho \in H^{1}(\Omega) \cap \mathcal{E}^{\prime}(\Omega)$ then, for all $x \in \mathcal{O}$,

$$
\Delta_{x} J(x)=2 \int_{\Omega}\left|\nabla_{y} \nabla_{x} \widetilde{\psi}\right|^{2} d y-2 \int_{\Omega} \widetilde{\rho}^{2} d y, \quad\left|\nabla_{y} \nabla_{x} \widetilde{\psi}\right|^{2}:=\sum_{j, k}\left|\frac{\partial^{2} \widetilde{\psi}}{\partial y_{j} \partial x_{k}}\right|^{2}
$$

Proof of Lemma 11.3. Differentiating (9.5) with respect to $x_{j}$ with $j=k$ yields

$$
\frac{\partial^{2} J}{\partial x_{k}^{2}}=2 \int_{\Omega} \frac{\partial}{\partial x_{k}}\left(\widetilde{\psi} \frac{\partial \widetilde{\rho}}{\partial y_{k}}\right) d y
$$

since $\nabla_{x} \widetilde{\rho}=\nabla_{y} \widetilde{\rho}$. Compute

$$
\frac{\partial}{\partial x_{k}}\left(\widetilde{\psi} \frac{\partial \widetilde{\rho}}{\partial y_{k}}\right)=\frac{\partial \widetilde{\psi}}{\partial x_{k}} \frac{\partial \widetilde{\rho}}{\partial y_{k}}+\widetilde{\psi} \frac{\partial^{2} \widetilde{\rho}}{\partial x_{k} \partial y_{k}}=\frac{\partial \widetilde{\psi}}{\partial x_{k}} \frac{\partial \widetilde{\rho}}{\partial y_{k}}+\widetilde{\psi} \frac{\partial^{2} \widetilde{\rho}}{\partial y_{k}^{2}}
$$

Since $\widetilde{\rho}$ is compactly supported in $\Omega$, integration by parts yields

$$
\int_{\Omega} \widetilde{\psi} \frac{\partial^{2} \widetilde{\rho}}{\partial y_{k}^{2}} d y=-\int_{\Omega} \frac{\partial \widetilde{\psi}}{\partial y_{k}} \frac{\partial \widetilde{\rho}}{\partial y_{k}} d y=\int_{\Omega} \widetilde{\rho} \frac{\partial^{2} \widetilde{\psi}}{\partial y_{k}^{2}} d y
$$

Therefore

$$
\frac{\partial^{2} J}{\partial x_{k}^{2}}=2 \int_{\Omega} \frac{\partial \widetilde{\rho}}{\partial y_{k}} \frac{\partial \widetilde{\psi}}{\partial x_{k}} d y+2 \int_{\Omega} \widetilde{\rho} \frac{\partial^{2} \widetilde{\psi}}{\partial y_{k}^{2}} d y
$$

To compute the first integrand, multiply the Poisson equation from (9.3) by $\partial \widetilde{\psi} / \partial x_{k}$ and use $\partial \widetilde{\rho} / \partial x_{k}=\partial \widetilde{\rho} / \partial y_{k}$ to find

$$
\begin{aligned}
\int_{\Omega} \frac{\partial \widetilde{\rho}}{\partial y_{k}} \frac{\partial \widetilde{\psi}}{\partial x_{k}} d y & =\int_{\Omega}\left(-\Delta_{y} \frac{\partial \widetilde{\psi}}{\partial x_{k}}\right) \frac{\partial \widetilde{\psi}}{\partial x_{k}} d y \\
& =\int_{\Omega}\left|\nabla_{y} \frac{\partial \widetilde{\psi}}{\partial x_{k}}\right|^{2} d y-\int_{\partial \Omega}\left(\nabla_{y} \frac{\partial \widetilde{\psi}}{\partial x_{k}}\right) \cdot \nu \frac{\partial \widetilde{\psi}}{\partial x_{k}} d S(y) .
\end{aligned}
$$

Since $\partial \widetilde{\psi} / \partial x_{k}$ is constant on the boundary one has

$$
\int_{\partial \Omega}\left(\nabla_{y} \frac{\partial \widetilde{\psi}}{\partial x_{k}}\right) \cdot \nu \frac{\partial \widetilde{\psi}}{\partial x_{k}} d S(y)=\left.\frac{\partial \widetilde{\psi}}{\partial x_{k}}\right|_{K} \int_{\partial \Omega}\left(\nabla_{y} \frac{\partial \widetilde{\psi}}{\partial x_{k}}\right) \cdot \nu d S(y)=0
$$

where the last condition from (9.3) is used for the last equality. Therefore

$$
\int_{\Omega} \frac{\partial \widetilde{\rho}}{\partial y_{k}} \frac{\partial \widetilde{\psi}}{\partial x_{k}} d y=\int_{\Omega}\left|\nabla_{y} \frac{\partial \widetilde{\psi}}{\partial x_{k}}\right|^{2} d y
$$


Summing (11.3) with respect to $k$ and using Poisson's equation (9.2) yields

$$
\Delta_{x} J(x)=\sum_{k=1}^{3} 2 \int_{\Omega}\left|\nabla_{y} \frac{\partial \widetilde{\psi}}{\partial x_{k}}\right|^{2} d y+2 \int_{\Omega} \widetilde{\rho} \Delta_{y} \widetilde{\psi} d y=2 \int_{\Omega}\left|\nabla_{y} \nabla_{x} \widetilde{\psi}\right|^{2} d y-2 \int_{\Omega} \widetilde{\rho}^{2} d y .
$$

This completes the proof of Lemma 11.3.

Lemma 11.4. The conclusion of Theorem 11.1 holds for $\rho \in H^{1}(\Omega) \cap \mathcal{E}^{\prime}(\Omega)$.

Proof. For smooth $\rho$, one has $G(x)=J(x)+C$ where $C$ is an additive constant. Therefore $\Delta G=\Delta J$. The first step proves $\Delta J \leq 0$. The second step analyses the case of equality, $\Delta J=0$.

Step I. Proof that $\Delta J \leq 0$. In view of (11.1) the Laplacian of $J$ appears as the difference of two positive terms. We prove that the first one is smaller or equal to the second. Following an idea of [4], the first term is given by the complementary energy minimization principle,

$$
\int_{\Omega}\left|\nabla_{y} \frac{\partial \widetilde{\psi}}{\partial x_{k}}\right|^{2} d y=\min _{\tau \in \mathcal{T}} \int_{\Omega}|\tau|^{2} d y
$$

where

$$
\mathcal{T}=\left\{\tau \in L^{2}\left(\Omega ; \mathbb{R}^{3}\right):-\operatorname{div}_{y} \tau=\frac{\partial \widetilde{\rho}}{\partial x_{k}} \text { in } \Omega, \quad \text { and, } \quad \int_{\partial \Omega} \tau \cdot \nu d S(y)=0\right\} .
$$

Take a test vector field $\tau=\nabla_{y} w_{k}$ with $w_{k} \in \dot{H}^{1}\left(\mathbb{R}^{3}\right)$ defined by

$$
-\Delta_{y} w_{k}=\frac{\partial \widetilde{\rho}}{\partial x_{k}} \text { in } \mathbb{R}^{3}
$$

Then $\int_{\partial \Omega} \tau \cdot \nu d S(y)=0$ since $\Delta_{y} w_{k}=0$ in $K$. It yields

$$
\int_{\Omega}\left|\nabla_{y} \frac{\partial \widetilde{\psi}}{\partial x_{k}}\right|^{2} d y \leq \int_{\Omega}\left|\nabla_{y} w_{k}\right|^{2} d y \leq \int_{\mathbb{R}^{3}}\left|\nabla_{y} w_{k}\right|^{2} d y
$$

But

so

$$
w_{k}=(-\Delta)^{-1} \frac{\partial \widetilde{\rho}}{\partial y_{k}}
$$

$$
\sum_{k} \frac{\partial w_{k}}{\partial y_{k}}=\left(-\Delta_{y}\right)^{-1} \Delta_{y} \widetilde{\rho}=-\widetilde{\rho}
$$

An integration by parts yields

$$
\sum_{k} \int_{\mathbb{R}^{3}}\left|\nabla_{y} w_{k}\right|^{2} d y=\sum_{k} \int_{\mathbb{R}^{3}} w_{k} \frac{\partial \widetilde{\rho}}{\partial y_{k}} d y=\int_{\mathbb{R}^{3}}|\widetilde{\rho}|^{2} d y .
$$


Therefore

$$
\sum_{k} \int_{\Omega}\left|\nabla_{y} \frac{\partial \widetilde{\psi}}{\partial x_{k}}\right|^{2} d y \leq \int_{\Omega}|\widetilde{\rho}|^{2} d y
$$

equivalent to $\Delta_{x} J(x) \leq 0$

Step II. When $\Delta J=0$ the external electric field is constant on a neighborhood of $K$. In case of equality $\Delta_{x} J(\bar{x})=0$ at a point $\bar{x}$. This is equivalent to equality in (11.6) which, in turn, is equivalent to a pair of equalities in (11.5). When the first inequality in (11.5) is an equality one has

$$
\nabla_{y} w_{k}(\bar{x}, \cdot)=\nabla_{y} \frac{\partial \widetilde{\psi}(\bar{x}, \cdot)}{\partial x_{k}} \quad \text { in } \Omega
$$

Equality in the second inequality in (11.5) implies that

$$
\nabla_{y} w_{k}(\bar{x}, \cdot)=0 \quad \text { in } K
$$

In particular the second equality implies that $w_{k}(\bar{x}, \cdot)$ satisfies the boundary conditions

$$
\left.w_{k}(\bar{x}, \cdot)\right|_{K}=\text { constant }, \quad \int_{\partial \Omega} \frac{\partial w_{k}(\bar{x}, \cdot)}{\partial \nu} d S(y)=0 .
$$

Thus, by uniqueness of the solution of (9.3),

$$
w_{k}(\bar{x}, \cdot)=\frac{\partial \widetilde{\psi}(\bar{x}, \cdot)}{\partial x_{k}} \quad \text { on } \Omega
$$

Since $K$ is connected, it belongs to a single connected component $\omega$ of $\mathbb{R}^{3} \backslash \operatorname{supp} \widetilde{\rho}(\bar{x}, \cdot)$. We know that $w_{k}(\bar{x}, \cdot)$ is constant inside $K$ and harmonic outside the support of $\widetilde{\rho}(\bar{x}, \cdot)$ and thus in $\omega \cup K$. Therefore, by analytic continuation there is a constant $v_{k}$ so that

$$
w_{k}(\bar{x}, y)=v_{k} \text { for } y \in \omega .
$$

Define the function $\widetilde{\phi} \in \dot{H}^{1}\left(\mathbb{R}^{3}\right)$ as the unique solution of (11.8) $-\Delta_{y} \widetilde{\phi}=\widetilde{\rho} \quad$ in $\quad \mathbb{R}^{3}, \quad$ so, $\quad w_{k}(x, y)=\frac{\partial \widetilde{\phi}}{\partial x_{k}}(x, y)=\frac{\partial \widetilde{\phi}}{\partial y_{k}}(x, y) \quad$ in $\mathbb{R}^{3}$.

The solution $\widetilde{\phi}(x, y)$ is a function of $x+y$. By $(11.8), \nabla_{y} \widetilde{\phi}(\bar{x}, y)=v$ for $y \in \omega$. Thus, there exists a constant $c_{1} \in \mathbb{R}$ such that

$$
\widetilde{\phi}(\bar{x}, y)=v \cdot y+c_{1} \text { for } y \in \omega .
$$

Therefore

$$
\widetilde{\phi}(x, y)=v \cdot(x+y-\bar{x})+c_{1}=v \cdot(x+y)+c_{2}, \quad \text { for } \quad x+y-\bar{x} \in \omega .
$$

Therefore,

$$
E_{k}=-\frac{\partial \widetilde{\phi}}{\partial y_{k}}=-w_{k}(x, y)=-v_{k} \quad \text { for } \quad y+x-\bar{x} \in \omega
$$


In case of equality, the external electric field is constant on a neighborhood of $K$.

Step III. When $\Delta J=0, J$ is affine. For $x$ sufficiently close to $\bar{x}, w_{k}$ satisfies

$$
-\Delta_{y} w_{k}=\frac{\partial \widetilde{\rho}}{\partial x_{k}} \quad \text { in } \quad \Omega,\left.\quad w_{k}(x, \cdot)\right|_{K}=\text { constant }, \quad \int_{\partial \Omega} \frac{\partial w_{k}(x, \cdot)}{\partial \nu} d S(y)=0 .
$$

By uniqueness of the solution of (9.3), for $x$ sufficiently close to $\bar{x}$,

$$
w_{k}(x, \cdot)=\frac{\partial \widetilde{\psi}}{\partial x_{k}}(x, \cdot) \quad \text { on } \Omega .
$$

Comparing (11.11) and (11.8) shows that there exists a function $\phi_{0}(y)$, which does not depend on $x$, such that, for any $x$ in a small neighborhood of $\bar{x}$,

$$
\widetilde{\psi}(x, y)=\widetilde{\phi}(x, y)+\phi_{0}(y) \quad \text { for any } y \in \Omega \text {. }
$$

Compute

$$
J(x)=\int_{\Omega} \widetilde{\rho} \widetilde{\psi} d y+\left.\sigma \widetilde{\psi}\right|_{K}=\int_{\Omega}\left(\widetilde{\rho} \widetilde{\phi}+\widetilde{\rho} \phi_{0}\right) d y+\left.\sigma \widetilde{\psi}\right|_{K} .
$$

In the right hand side of (11.13) the first term is independent of $x$, by invariance by translation of the integral. The third term is affine in $x$ because $\widetilde{\psi}$ is constant with respect to $y$ on $K$ and, by virtue of (11.12) and (11.9) is affine in $x$ on $\partial K$. The second term is, by integration by parts,

$\int_{\Omega} \widetilde{\rho} \phi_{0} d y=-\int_{\Omega} \Delta_{y} \widetilde{\phi} \phi_{0} d y=-\int_{\partial K} \partial_{\nu} \widetilde{\phi} \phi_{0}+\int_{\partial K} \partial_{\nu} \phi_{0} \widetilde{\phi}-\int_{\Omega} \widetilde{\phi} \Delta_{y} \phi_{0} d y=a \cdot x+b$, since $\widetilde{\phi}$ is affine in $x$ in a neighborhood of $K$ and

$$
\Delta_{y} \phi_{0}=\Delta_{y} \widetilde{\psi}-\Delta_{y} \widetilde{\phi}=0 \quad \text { in } \Omega \text {. }
$$

Therefore, $J(x)=a^{\prime} \cdot x+b^{\prime}$ for $x$ close to $\bar{x}$.

Proof of Theorem 11.1. The values of $G(x)$ depend only on the values of $\psi$ on a neighborhood of $K(\underline{x})$. Given $\rho$ choose $0<\epsilon<\operatorname{dist}(\operatorname{supp} \rho, K(\underline{x}))$. Choose $\zeta^{\epsilon} \in C^{\infty}\left(\mathbb{R}^{3}\right)$ so that $\zeta^{\epsilon}(y)=1$ for $\operatorname{dist}(y, \operatorname{supp} \rho)>\epsilon$ and $\zeta^{\epsilon}(y)=0$ for $\operatorname{dist}(y, \operatorname{supp} \rho)<\epsilon / 2$. Denote by $\psi$ the electrostatic potential of $\rho$ and $\psi^{\epsilon}:=\zeta \psi$ that of $\rho^{\epsilon}=-\Delta \psi^{\epsilon}$. Then the charges $\rho^{\epsilon}$ are smooth and supported in an $\epsilon$-neighborhood of $\operatorname{supp} \rho$.

The Lemma 11.4 implies that the corresponding $\Delta G^{\epsilon}(x) \leq 0$ on a neighborhood of $\underline{x}$. Since $\psi$ and $\psi^{\epsilon}$ are equal on a neighborhood of $K(\underline{x})$, part $\mathbf{i}$ of Proposition 10.2 implies that $G^{\epsilon}(x)$ is equal to $G(x)$ on a neighborhood of $\underline{x}$. The proof is complete.

Theorem 11.5. Suppose that $K$ a connected perfectly conducting body. If $K(\underline{x})$ is an equilibrium position in the field of external charges $\rho \in \mathcal{E}^{\prime}\left(\mathbb{R}^{3}\right)$ with $K(\underline{x}) \cap \operatorname{supp} \rho=\varnothing$, then the equilibrium is unstable.

The proof, entirely analogous to the proof of Theorem 7.1, is omitted. 
Remark 11.6. A renormalized energy $G$ in the case of dielectrics is defined by expressing $\psi=\phi_{\text {ext }}+\zeta$ as in Proposition 3.2 and the energy as

$$
J=-\frac{1}{2} \int_{\mathbb{R}^{3}}(\rho+q)\left(\phi_{\text {ext }}+\zeta\right) d y:=-\frac{1}{2} \int_{\mathbb{R}^{3}} \rho \phi_{\text {ext }} d y+G .
$$

Analogues of Proposition 10.2 and its Corollary are true for this renormalized energy. A regularization argument as in the proof of Theorem 11.1 extends Theorem $\% .1$ to external charges $\rho \in \mathcal{E}^{\prime}\left(\mathbb{R}^{3}\right)$ with $K(\underline{x}) \cap \operatorname{supp} \rho=\varnothing$.

Acknowledgments. G.A. is a member of the DEFI project at INRIA Saclay Ile-deFrance.

\section{REFERENCES}

[1] V. Arnold, Mathematical developments arising from Hilbert problems, in Proceedings of Symposia in Pure Mathematics, F. Browder, ed., Amer. Math. Soc., Providence, R.I., 1976.

[2] V. Arnold, Mathematical Methods of Classical Mechanics, Graduate Texts in Mathematics Vol. 60, Springer-Verlag, 1997

[3] R.J. Duffin, Free suspension and Earnshaw's theorem, Arch. Rational Mech. Anal. 14(1963) 261-163.

[4] R.J. Duffin, The potential energy of an electric charge, Arch. Rational Mech. Anal. 15(1964) 305-310.

[5] R.J. Duffin and A. Shild, The potential energy of an electric charge is a superharmonic function, Arch. Rational Mech. Anal. 25(1967) 156-158.

[6] S. Earnshaw, On the nature of the molecular forces which regulate the constitution of the luminiferous ether, Trans. Cambridge Phil. Soc. 7, 97114 (1842)

[7] A. Henrot and M. Pierre, Variation et optimisation de formes, une analyse géométrique, Springer (2005).

[8] V. Kozlov, Asymptotic solutions of equations of classical mechanics, J. Appl. Math. Mech. 46 (1982) 454-457.

[9] V. Kozlov, Asymptotic motions and the inversion of the Lagrange-Dirichlet theorem, J. Appl. Math. Mech. 50 (1987) 719-725.

[10] M. Laloy and K. Peiffer, On the instability of equilibrium when the potential has a non-strict local minimum, Arch. Rational Mech. Anal. 78 (1982) 213-222.

[11] J. C. Maxwell, A Treatise on Electricity and Magnetism Vol. I., (From the 1891 ed.) Dover Publ. 1954.

[12] I. Newton, Mathematical Principles of Natural Philosophy, B. Cohen translator, Univ. California Press, 2016.

[13] P. Painlevé. Sur la stabilité de l'équilibre. C.R. Acad. Sci. Paris. Ser. A-B 135. IS55 (1904)

[14] V.P. Palamodov, Stability of equilibrium in a potential field. Funkts. Anal. Prilozh. 11, No. 4, 4255 (1977). Engl. transl.: Funct. Anal. Appl. 11, (1978) 277289.

[15] V.P. Palamodov, Stability of motion and algebraic geometry, in Kozlov,V.V. ed., Dynamical Systems in Classical Mechanics, Transl., Ser. 2, Am. Math. Soc. 168(25) (1995), 5-20.

[16] J. Rauch, Earnshaw's theorem in electrostatics and a conditional converse to Dirichlet's Theorem, Séminaire Laurent Schwartz, École Polytechnique, January 2014.

[17] S. Taliaferro, Stability for two dimensional analytic potentials, J. Differential Equations 35 (1980) 248-265.

[18] S. Taliaferro, Instability of an equilibrium in a potential field. Arch. Rational Mech. Anal. 109 no.2 (1990) 183-194.

[19] A. Wintner, The Analytical Foundations of Celestial Mechanics, Princeton University Press, Princeton N.J., (1941). 